\title{
THE SUBGROUPS OF A FREE PRODUCT OF TWO GROUPS WITH AN AMALGAMATED SUBGROUP( $\left.{ }^{1}\right)$
}

\author{
BY \\ A. KARRASS AND D. SOLITAR
}

\begin{abstract}
We prove that all subgroups $H$ of a free product $G$ of two groups $A, B$ with an amalgamated subgroup $U$ are obtained by two constructions from the intersection of $H$ and certain conjugates of $A, B$, and $U$. The constructions are those of a tree product, a special kind of generalized free product, and of a Higman-NeumannNeumann group. The particular conjugates of $A, B$, and $U$ involved are given by double coset representatives in a compatible regular extended Schreier system for $G$ modulo $H$. The structure of subgroups indecomposable with respect to amalgamated product, and of subgroups satisfying a nontrivial law is specified. Let $A$ and $B$ have the property $P$ and $U$ have the property $Q$. Then it is proved that $G$ has the property $P$ in the following cases: $P$ means every f.g. (finitely generated) subgroup is finitely presented, and $Q$ means every subgroup is f.g.; $P$ means the intersection of two f.g. subgroups is f.g., and $Q$ means finite; $P$ means locally indicable, and $Q$ means cyclic. It is also proved that if $N$ is a f.g. normal subgroup of $G$ not contained in $U$, then $N U$ has finite index in $G$.
\end{abstract}

1. Introduction. In the case of a free product $G=A * B$, the structure of a subgroup $H$ may be described as follows (see $[9,4.3])$ : there exist double coset representative systems $\left\{D_{\alpha}\right\},\left\{D_{\beta}\right\}$ for $G \bmod (H, A)$ and $G \bmod (H, B)$ respectively, and there exists a set of elements $t_{1}, t_{2}, \ldots$ (possibly empty) such that

(1) $H=\operatorname{gp}\left(t_{1}, t_{2}, \ldots, D_{\alpha} A D_{\alpha}^{-1} \cap H, \ldots, D_{\beta} B D_{\beta}^{-1} \cap H, \ldots\right)$;

(2) $t_{1}, t_{2}, \ldots$ freely generate a free group $F$;

(3) $D_{\alpha} A D_{\alpha}^{-1} \cap H, \ldots, D_{\beta} B D_{\beta}^{-1} \cap H, \ldots$ generate their free product $S$; and

(4) $H$ is the free product of $F$ and $S$.

The structure of a subgroup $H$ of a free product $G=(A * B ; U)$ with an amalgamated subgroup has an analogous (although more complicated) description: There exist double coset representatives $\left\{D_{\alpha}\right\},\left\{D_{\beta}\right\}$ for $G \bmod (H, A)$ and $G \bmod$ $(H, B)$ respectively, and there exists a set of elements $t_{1}, t_{2}, \ldots$ such that

Received by the editors September 2, 1969.

AMS Subject Classifications. Primary 2052, 2010, 2027; Secondary 2018, 2008, 2054.

Key Words and Phrases. Amalgamated products, generalized free products, tree products, subgroup structure, indecomposable subgroups, presentations, Schreier system, ReidemeisterSchreier theory, compatible regular extended Schreier system, Higman-Neumann-Neumann groups, HNN groups, finitely generated intersection property, finitely presented subgroups, Kuroš subgroup theorem, double-ended cosets, locally indicable groups, simply decomposable groups.

(1) This research was supported by a grant from the National Research Council of Canada. Copyright (C) 1970, American Mathematical Society 
(5) $H=\operatorname{gp}\left(t_{1}, t_{2}, \ldots, D_{\alpha} A D_{\alpha}^{-1} \cap H, \ldots, D_{\beta} B D_{\beta}^{-1} \cap H, \ldots\right)$;

(6) $t_{1}, t_{2}, \ldots$ freely generate a free group $F$;

(7) $D_{\alpha} A D_{\alpha}^{-1} \cap H, \ldots, D_{\beta} B D_{\beta}^{-1} \cap H, \ldots$ generate a generalized free product $S$ with amalgamated subgroups $D_{\alpha} U D_{\alpha}^{-1} \cap H, \ldots, D_{\beta} U D_{\beta}^{-1} \cap H, \ldots$ (where $S$ is a special type of generalized free product which we call a "tree product"); and

(8) $H$ is the free product of the groups $T_{i}=\operatorname{gp}\left(t_{i}, S\right)$ with the subgroup $S$ amalgamated.

If $U=1, T_{i}$ will be the free product of $\mathrm{gp}\left(t_{i}\right)$ and $S$, so that (8) reduces to (4). Indeed for any $U$, the subgroup $T_{i}$ is a free product of $\operatorname{gp}\left(t_{i}, K_{i} U K_{i}^{-1} \cap H\right)$ and the group $S$ with the $\mathrm{gp}\left(K_{i} U K_{i}^{-1} \cap H, t_{i} K_{i} U K_{i}^{-1} t_{i}^{-1} \cap H\right)$ amalgamated where $K_{i}$ is some element of $G$. Hence, if $U=1$, or more generally, if $H$ has trivial intersection with each conjugate of $U$, then (7) reduces to (3), $T_{i}=\mathrm{gp}\left(t_{i}\right) * S$, so that (8) reduces to (4), and so $H$ is the free product of a free group and subgroups of conjugates of the factors. This result is implicit in H. Neumann [13].

Now (7) refers to the notion of a generalized free product of groups with amalgamated subgroups. This construction was introduced by $\mathrm{H}$. Neumann [12] for the purpose of describing the structure of the subgroups of $G=(A * B ; U)$; in fact, $H$. Neumann showed that this construction can be used to describe the subgroups of a generalized free product with amalgamated subgroups. However, for the special case $G=(A * B ; U)$, one can describe its subgroups using a simpler construction than the generalized free product, viz., a "tree product", which we now proceed to define.

Let $\left\{A_{i}\right\}$ be a collection of groups and suppose that with certain pairs of groups $A_{i}, A_{j}$ there is associated an isomorphism $\theta_{i j}$ from a subgroup $U_{i j}$ of $A_{i}$ onto a subgroup $U_{j i}$ of $A_{j}$, such that $\theta_{j i}=\theta_{i j}^{-1}$. Then the partial generalized free product of the factors $A_{i}$ is the group $G$ having the presentation obtained by presenting each of the amalgamated products $\left(A_{i} * A_{j} ; U_{i j}=U_{j i}\right)$ with $U_{i j}$ and $U_{j i}$ amalgamated under $\theta_{i j}$ (using a fixed presentation for each $A_{i}$ ), and then taking the union of these presentations. (It is easily shown that $G$ is independent of the particular presentation used for $A_{i}$ and the particular set of generators used for $U_{i j}$.)

With each partial generalized free product $G$ we associate a linear graph each of whose vertices corresponds to a factor $A_{i}$ and each of whose edges joins two vertices $A_{i}$ and $A_{j}$ if $\theta_{i j}$ (and hence $\theta_{j i}$ ) exists. If this graph is a tree, then we call $G$ a tree product of the factors $A_{i}$ (with the subgroups $U_{i j}$ and $U_{j i}$ amalgamated under $\theta_{i j}$ ), and denote $G$ by

or by

$$
\Pi^{*}\left(A_{i} ; U_{j k}=\theta_{j k}\left(U_{j k}\right)\right)
$$

$$
\Pi^{*}\left(A_{i} ; U_{j k}=U_{k j}\right)
$$

when specific $\theta_{j k}$ are implicit.

We shall show that the tree product of the $A_{i}$ contains each of the factors $A_{i}$ in the natural way, and that two factors $A_{i}$ and $A_{j}$ which are neighbors, i.e., joined 
by an edge, generate their amalgamated product. More generally, the factors in any subtree generate their tree product.

[Note that the definition, as given by H. Neumann [12], of the generalized free product of groups $\left\{A_{i}\right\}$ with amalgamated subgroups is the partial generalized free product $G$ as defined above with the additional requirements that the isomorphisms $\theta_{i j}$ exist for all pairs $i \neq j$ and that each factor $A_{i}$ must be naturally contained as a subgroup of $G$. Although the partial generalized free product exists even if $\theta_{i j}$ is specified for all pairs $i \neq j$, the generalized free product may not exist. Moreover, even if it exists, in general the subgroup generated by two factors is not their amalgamated product.]

The groups $T_{i}$ in (8) and the group $H$ itself are special cases of groups which we call "HNN groups" (used by Higman, Neumann, and Neumann in [6]) defined in $\S 4$. The structure of a subgroup $H$ of $G=(A * B ; U)$ has a simple description in terms of tree products and HNN groups (see Theorem 5).

Next we describe some of the applications we have obtained from the subgroup theorem.

G. Baumslag [3] proved that if $G=(A * B ; U)$ where $A, B$ are free and $U$ is maximal cyclic in $A$ and in $B$, then any two-generator subgroup of $G$ is free; on the other hand, $n$-generator subgroups for $n>2$ need not be free. He conjectured, however, that all finitely generated subgroups are finitely related. We establish the following generalization of his conjecture:

Let $G=(A * B ; U)$ where $A, B$ have the property that their finitely generated subgroups are finitely related and suppose every subgroup of $U$ is finitely generated. Then every finitely generated subgroup of $G$ is finitely related (see Theorem 8 ). (If $U$ does not satisfy the maximal condition then $G$ may have finitely generated subgroups which are not finitely related.)

B. Baumslag [1] extended theorems of Schreier [14] and Howson [7] on finitely generated subgroups of free groups to free products. He proved that a finitely generated normal subgroup $(\neq 1)$ of a free product is of finite index; and if $A, B$ have the finitely generated intersection property (i.e., the intersection of any two finitely generated subgroups is finitely generated), then $G=A * B$ has the finitely generated intersection property. We generalize his results as follows:

Let $G=(A * B ; U)$ and let $H$ be a finitely generated normal subgroup of $G$ not contained in $U$. Then the subgroup $H U$ is of finite index in $G$; in particular, $H$ is of finite index in $G$ iff $U \cap H$ is of finite index in $U$ (see Theorem 10).

Let $G=(A * B ; U)$ where $A, B$ have the finitely generated intersection property and $U$ is finite. Then $G$ has the finitely generated intersection property (see Theorem 11). (On the other hand if $U$ is infinite, even infinite cyclic, then $G$ need not have the finitely generated intersection property.)

D. I. Moldavanski [10] proved that an abelian subgroup of $G=(A * B ; U)$ is either contained in a conjugate of $A$ or $B$, or is the ascending union of subgroups of conjugates of $U$, or is the direct product of a subgroup of a conjugate of $U$ 
and an infinite cyclic group. Now an abelian group $H$ is, in particular, indecomposable with respect to amalgamated product (i.e., $H=(C * D ; V)$ implies $C=V$ or $D=V)$. We extend his result to the following:

Let $G=(A * B ; U)$ and let $H$ be an indecomposable (with respect to amalgamated product) subgroup of $G$. Then $H$ is contained in a conjugate of $A$ or $B$, or $H$ is the countable ascending union of subgroups of conjugates of $U$, or $H$ is generated by $a$ subgroup of a conjugate of $U$ and an additional element not in a conjugate of a factor (see Theorem 6).

Moreover, if $H$ satisfies an identity not satisfied by the infinite dihedral group (the free product of two groups of order two), then $H$ is indecomposable and so has the structure indicated above (indeed one can be more explicit, see Theorem 7); if $H$ satisfies a nontrivial identity which holds in the infinite dihedral group, then in addition to the three possibilities above, $H$ may be the free product of two conjugates $C$ and $D$ of subgroups of $A$ or $B$ with a subgroup of a conjugate of $U$ amalgamated (and this amalgamated subgroup is of index two in $C$ and $D$ ) (see Theorem 7).

An "indicable group" is one having an infinite cyclic factor group. (G. Higman [4] showed that the group ring of a locally indicable group has no zero divisors if the coefficient ring has none.) We show that if $A, B$ are locally indicable and $U$ is cyclic, then $G$ is locally indicable (see Theorem 9).

More detailed descriptions and other applications of the above results are also included.

Finally, we wish to mention that this paper was inspired by the work of $\mathrm{H}$. Neumann [13] on the structure of the subgroups of an amalgamated product; it arose in an attempt to make her results more explicit for the case of two factors.

2. Notations. The following notations will be used for the free product $G$ of two groups $A, B$ with the subgroup $U$ generated by $u_{1}, u_{2}, \ldots$ in $A$ and the subgroup $V$ generated by $v_{1}, v_{2}, \ldots$ in $B$ amalgamated under an isomorphism $\varphi$ which carries $u_{i}$ into $v_{i}$ :

$$
(A * B ; U=V), \quad\left(A * B ; u_{i}=v_{i}\right), \quad(A * B ; U=\varphi(U)) .
$$

Moreover, if $\varphi$ is the identity mapping on $U$, then we use $(A * B ; U)$ or $A *_{U} B$. Similar notations are used for amalgamated or tree products involving more than two factors, e.g., $A_{1} *_{U_{12}} A_{2} *_{U_{23}} A_{3}$ is the tree product of $A_{1}, A_{2}, A_{3}$, with the subgroup $U_{12}$ amalgamated between $A_{1}$ and $A_{2}$ and the subgroup $U_{23}$ amalgamated between $A_{2}$ and $A_{3}$; the notation $\Pi^{*}\left(A_{i} ; U_{j k}=U_{k j}\right)$ for a tree product was introduced previously; $\Pi^{*}\left(A_{i} ; U\right)$ is the free product of the groups $A_{i}$ with the common subgroup $U$ amalgamated. (Note that $\Pi^{*}\left(A_{i} ; U\right)$ may be viewed as a tree product in many ways; indeed, construct any tree using the factors $A_{\mathfrak{i}}$ as vertices, and let the subgroup $U$ correspond to each edge.)

Let $K$ be a group with presentation

$$
\left\langle k_{1}, k_{2}, \ldots ; R_{1}, R_{2}, \ldots\right\rangle
$$


and let $\varphi$ be an isomorphism of a subgroup $U$ into $K$ where $U$ is generated by $\left\{u_{i}\left(k_{j}\right)\right\}$, and $v_{i}\left(k_{j}\right)=\varphi\left(u_{i}\right)$. Then the group

is the group

$$
\left\langle x, K ; \operatorname{rel} K, x U x^{-1}=\varphi(U)\right\rangle
$$

$$
\left\langle x, k_{1}, k_{2}, \ldots ; R_{1}, R_{2}, \ldots, x u_{1} x^{-1}=v_{1}, x u_{2} x^{-1}=v_{2}, \ldots\right\rangle .
$$

We define the group

$$
\left\langle x_{1}, x_{2}, \ldots, K ; \operatorname{rel} K, x_{1} U_{1} x_{1}^{-1}=\varphi_{1}\left(U_{1}\right), x_{2} U_{2} x_{2}^{-1}=\varphi_{2}\left(U_{2}\right), \ldots\right\rangle
$$

where $\varphi_{i}$ is an isomorphism of the subgroup $U_{i}$ into $K$ in a similar manner.

Let $H$ be a fixed subgroup of $G$; if $X<G$ and $g \in G$, then the subgroup $g X g^{-1}$ $\cap H$ will be denoted by " $X_{H}^{g}$ ".

The notation $\operatorname{HNN}\left(K,\left\{\varphi_{i}\right\}\right)$ is explained in $\S 4$.

Notations for various types of coset representative systems will be found in $\S 5$.

3. Tree products.

LEMMA 1. A graph is a tree if there exists a function which assigns to each vertex a nonnegative integer (called the "level" of the vertex) such that

(1) the vertices of level zero together with the edges joining them form a tree, and

(2) each vertex of level $n>0$ is joined by an edge to exactly one vertex of level less than or equal to $n$, and the level of this vertex is $n-1$.

Conversely, if the graph is a tree, such a function exists whose vertices of level zero are a given subtree.

Proof. Suppose first such a function $\lambda(w)$ exists. Clearly there is a path joining any vertex to a vertex of level zero; since any two vertices of level zero can be joined by a path, the graph is connected. Moreover, if a closed path $w=w_{1}$, $w_{2}, \ldots, w_{r}=w$ has maximum level $n>0$ on its vertices, we may assume $w$ has level $n$. By (2), $\lambda\left(w_{1}\right)>\lambda\left(w_{2}\right)$ and $\lambda\left(w_{r}\right)>\lambda\left(w_{r-1}\right)$, and so by $(2), w_{2}=w_{r-1}$, so that the closed path is not simple. Hence the given graph must be a tree.

Conversely, suppose the given graph is a tree. Choose the vertices of any subtree to have level zero, and define the level of any vertex to be the minimum length (number of edges) of a simple path joining the vertex to a vertex of level zero. It is easy to show that conditions (1) and (2) hold.

Definition. If the vertices of a graph $\Gamma$ are partitioned into subsets $\Gamma_{i}$, then the graph arising from $\Gamma$ by contracting the $\Gamma_{i}$ to vertices is the graph whose vertices are the $\Gamma_{i}$ and whose edges are the edges of $\Gamma$ which join vertices of $\Gamma_{i}$ and $\Gamma_{j}$, $i \neq j$.

It is easy to see that if $\Gamma$ is a tree and the $\Gamma_{i}$ are subtrees, then the graph which arises by contracting the $\Gamma_{i}$ to vertices is itself a tree.

DEFINITION. Let

$$
\left\langle h_{1}, h_{2}, \ldots ; S_{1}\left(h_{j}\right), S_{2}\left(h_{j}\right), \ldots\right\rangle
$$


be a presentation for a group $H$, and let

$$
\left\langle g_{1}, g_{2}, \ldots, h_{1}, h_{2}, \ldots ; R_{1}\left(g_{i}, h_{j}\right), R_{2}\left(g_{i}, h_{j}\right), \ldots, S_{1}\left(h_{j}\right), S_{2}\left(h_{j}\right), \ldots\right\rangle,
$$

where each $R_{k}\left(g_{i}, h_{j}\right)$ involves some $g_{i}$, be a presentation for a group $G$. Then we say that $H$ is a subgroup of $G$ in the natural way (relative to the given presentations for $H$ and $G$ ) if the subgroup of $G$ generated by $h_{1}, h_{2}, \ldots$ has the presentation (1).

If $H, G$ are groups which have presentations defined in terms of presentations for other groups $A, B, C, \ldots$, then when we say " $H$ is a subgroup of $G$ in the natural way" without specifying presentations for $H, G$, we intend that the presentations of $A, B, C, \ldots$ used in constructing a presentation for $H$ be used in constructing a presentation for $G$. For example, if $H$ is the free product $A * B$, and $G$ is the free product $A * B * C$, then $H$ is a subgroup of $G$ in the natural way.

THEOREM 1. Let $G$ be a tree product $\Pi^{*}\left(A_{i} ; U_{j k}=U_{k j}\right)$.

(1) Then each vertex $A_{i}$ is a subgroup of $G$ in the natural way.

(2) Moreover, the tree product of a subtree is a subgroup of $G$ in the natural way.

(3) Finally, if the vertices of the graph of $G$ are partitioned into subtrees whose tree products are $G_{1}, G_{2}, \ldots$ and these subtrees are contracted to vertices, then $G$ is the tree product of the vertices $G_{1}, G_{2}, \ldots$ of the resulting tree.

Proof. Consider first the special case where there is a fixed vertex of $G$, say $A_{1}$, to which all the other vertices $A_{j}$ of $G$ are joined. (We then say $G$ is a "tree product of the $A_{i}$ with amalgamations from one factor".) If the subgroups $U_{1 j}$ coincide, $G$ is simply the free product of the $A_{i}$ with a single subgroup amalgamated, and in this case it is well known that each of the $A_{i}$ is a subgroup of $G$ in the natural way. If the $U_{1 j}$ do not all coincide, we first form the groups

$$
G_{1 j}=\left(A_{j} * A_{1} ; U_{j 1}=U_{1 j}\right)
$$

which contain $A_{j}$ and $A_{1}$ in the natural way; we then form $\Pi^{*}\left(G_{1 j} ; A_{1}\right)$ which has the same presentation as $G$. Hence $G_{1 j}$ and therefore all the vertices $A_{i}$ are contained in $G$ in the natural way.

To prove (1) in general, select any vertex, say $A_{0}$, to have level zero, and define the level of any other vertex as in the proof of Lemma 1 . Then the vertices of level $\leqq n$ together with the edges joining them form a connected subgraph of $G$ and hence a subtree. Let $L_{n}$ be the tree product of the vertices of level $\leqq n$. We show by induction on $n$ that each vertex of $L_{n}$ is a subgroup of $L_{n}$ in the natural way, and that $L_{n}$ is a subgroup of $L_{n+1}$ in the natural way.

Since $L_{0}=A_{0}$, the case $n=0$ is trivial. Suppose that each vertex of $L_{n}$ is a subgroup of $L_{n}$ in the natural way. Now each vertex $A_{j}$ of level $n+1$ can be joined to a unique vertex of $L_{n}$ by an edge corresponding to some subgroup $U_{j}$; and $L_{n+1}$ is just the tree product of the $A_{j}$ of level $n+1$ and $L_{n}$ with amalgamations from the one factor $L_{n}$. Hence $L_{n}$ and all the vertices of level $\leqq n+1$ are subgroups of $L_{n+1}$ in the natural way. 
Since $G$ has a presentation which is the union of presentations for the $L_{n}$, and $L_{n}$ is a subgroup of $L_{n+1}$ in the natural way, it follows that $L_{n}$ and therefore each vertex of $L_{n}$ is a subgroup of $G$ in the natural way (see, for example, exercise 18 on p. 33 of [9]). This proves (1).

To prove (2), let $L$ be the tree product of a subtree of $G$. Define a level function on the vertices of $G$ as in the proof of Lemma 1, using $L$ as the subtree of vertices of level zero. If again $L_{n}$ is the tree product of the vertices of level $\leqq n$, the same proof as above shows that $L$ is a subgroup of $G$ in the natural way.

Finally, to prove (3) note that each of the vertices of $G_{i}$ is a subgroup of $G_{i}$ in the natural way. Clearly a presentation for $G$ results when the tree product of the $G_{i}$ is formed.

Definition. A group $G$ is indecomposable (with respect to amalgamated product) if $G=(A * B ; U)$ implies $A=U$ or $B=U$.

Clearly, if $G$ is indecomposable and $G=\Pi^{*}\left(A_{i} ; U\right)$, then all $A_{i}$ except possibly one of them must equal $U$.

THEOREM 2. Let $G$ be a tree product

$$
\Pi^{*}\left(A_{i} ; U_{j k}=U_{k j}\right),
$$

and let $\lambda$ be a level function for the associated tree. If $G$ is indecomposable, then either $G$ equals one of its factors $A_{i}$ or $G$ is the ascending union of a chain of amalgamated subgroups corresponding to the edges of an infinite path determined by distinct vertices $A_{i_{0}}, A_{i_{1}}, \ldots, A_{i_{j}}, \ldots$ where $\lambda\left(A_{i_{j}}\right)=j$ for each $j$ or $\lambda\left(A_{i_{j}}\right)=0$ for each $j$.

Proof. Suppose that $G$ is not equal to any $A_{i}$.

We first consider the case when $G$ has only one vertex of level zero, say $A_{0}$. Let $\left\{A_{j}\right\}, j \in J_{0}$, be the vertices of $G$ which are joined to $A_{0}$ by an edge. If the vertex $A_{0}$ is deleted from the graph of $G$ each of the resulting components will be determined by exactly one $A_{j}, j \in J_{0}$; let $C_{0 j}$ be the tree product of the component containing $A_{j}$, and let

$$
G_{0 j}=\left(C_{0 j} * A_{0} ; U_{0 j}\right)
$$

Then by Theorem 1, $G$ is the tree product of the $C_{0 j}$ and $A_{0}$ with amalgamations from the factor $A_{0}$; hence

$$
G=\Pi_{j}^{*}\left(G_{0 j} ; A_{0}\right), \quad j \in J_{0} .
$$

Since $G$ is indecomposable, all but one of the factors $G_{0 j}$ must equal $A_{0}$; since $G$ is not one of its vertices, some $G_{0 j}$, say $G_{01}$, is different from $A_{0}$. Hence $G=G_{01}$ $=\left(C_{01} * A_{0} ; U_{01}\right)$, and so $A_{0}=U_{01}<A_{1}<C_{01}=G$. Moreover, each vertex of $G$ is either a subgroup of $A_{0}$ or is a vertex of $C_{01}$. Since $A_{0}$ is the only vertex of level zero in $G, A_{1}$ must have level one, and all other vertices of $C_{01}$ have level at least two.

Repeating the above argument using the graph of $C_{01}$ in place of the graph of 
$G$ and $A_{1}$ in place of $A_{0}$, we obtain $A_{0}=U_{01}<A_{1}=U_{12}<A_{2}<C_{12}=G, G=$ $\left(C_{12} * A_{1} ; U_{12}\right)$, and every vertex of $G$ is either contained in $A_{1}$ or is a vertex of $C_{12}$. Since $A_{1}$ is the only vertex of level at most one in $C_{01}, A_{2}$ must have level two, and all the other vertices of $C_{12}$ have level at least three.

Continuing in this way, we obtain an infinite sequence of distinct vertices

$$
A_{0}=U_{01}<A_{1}=U_{12}<A_{2}=U_{23}<A_{3}=U_{34}<A_{4} \cdots ;
$$

and $G=\left(C_{n, n+1} * A_{n} ; U_{n, n+1}\right)$; moreover every vertex of $G$ is either a subgroup of $A_{n}$ or is a vertex of $C_{n, n+1}$. Also $A_{n}$ is the only vertex of level at most $n$ in $C_{n-1, n}$, so that $A_{n+1}$ has level $n+1$ and all the other vertices of $C_{n, n+1}$ have level at least $n+2$.

Since every vertex of $G$ has a finite level, each vertex is a subgroup of some $A_{n}=U_{n, n+1}$; consequently $G$ is the ascending union of the amalgamated subgroups $U_{n, n+1}$ where $n=0,1,2, \ldots$ This completes the argument for the first case.

Suppose now that $G$ has several vertices of level zero. Let $L_{0}$ be the tree product of the subtree of $G$ determined by the vertices of level zero. Contract $L_{0}$ to a vertex; then $G$ is the tree product of a tree with a unique vertex $L_{0}$ of level zero. Since $G$ is indecomposable and not equal to any $A_{i}$, by the first case either $G=L_{0}$ or $G$ is the ascending union.

$$
L_{0}=U_{01}<A_{1}=U_{12}<A_{2}<\cdots .
$$

In the latter case, if $A_{0}$ is the vertex of $L_{0}$ joined to $A_{1}$ by an edge, $G$ is the ascending union

$$
A_{0}=U_{01}<A_{1}=U_{12}<A_{2} \cdots .
$$

If $G=L_{0}$, we may assign new levels to the vertices of $L_{0}$ so that there is a single vertex of new level zero. The first case then shows that $G$ is again an ascending union

$$
A_{0}=U_{01}<A_{1}=U_{12}<A_{2} \cdots
$$

where now each $A_{n}$ has old level zero.

Definition. A group $G$ is simply decomposable if whenever $G=A *_{U} B *_{V} C$, then $A=U$ or $C=V$ or $B=U=V$.

Clearly, if $G=\Pi^{*}\left(A_{i} ; U\right)$ and $G$ is simply decomposable, then all but possibly two of the $A_{i}$ are equal to $U$.

Suppose a group $G$ satisfies a nontrivial identity; then $G$ is simply decomposable. For, if $G=(A * B ; U)$ with $A \neq U \neq B$, then $U$ has index two in both $A$ and $B$ (indeed if $1, a_{1}, a_{2}$ determine different cosets of $U$ in $A$ and $b \in B-U$, then $a_{1}^{-1} b a_{2}$ and $b^{-1} a_{1}^{-1} b a_{2} b$ generate a free subgroup of rank two, contrary to $G$ satisfying a nontrivial identity). But if $G=A *_{U} B *_{V} C$ where $A \neq U, C \neq V$, and $B \neq V$, then $G=A *_{U}\left(B *_{V} C\right)$, and $U$ would have infinite index in $B *_{V} C$. Thus $G$ is simply decomposable. 
THEOREM 3. Let $G$ be a tree product

$$
\Pi^{*}\left(A_{i} ; U_{j k}=U_{k j}\right),
$$

and let $\lambda$ be a level function for the associated tree. If $G$ is simply decomposable, then $G$ equals one of its factors $A_{i}$, or $G$ is an ascending union of a chain of amalgamated subgroups as in Theorem 2 , or $G$ is the free product $\left(A_{i} * A_{j} ; U\right)$ of two of its factors $A_{i}, A_{j}$ with a subgroup $U$ amalgamated where $U$ corresponds to each edge in the simple path joining $A_{i}$ to $A_{j}$ and all the other vertices of the path are equal to $U$.

Proof. Suppose $G$ is not equal to any $A_{i}$.

As before we first consider the case when $G$ has only one vertex of level zero. We start to construct the sequence $A_{0}, A_{1}, A_{2}, \ldots$ as in the proof of Theorem 2 and note that we only used the indecomposability of $G$ to infer that when $A_{n}$ was deleted from the graph of $C_{n-1, n}$ only one of the resulting components, namely, $C_{n, n+1}$, was not a subgroup of $A_{n}$; and also to infer that $A_{n}$ was a subgroup of $U_{n, n+1}$. Hence we can show that $G$ is an ascending union of a chain of amalgamated subgroups as in Theorem 2 unless for some $n$ one of the following occurs when $A_{n}$ is deleted from the graph of $C_{n-1, n}$ : at least two of the resulting components are not subgroups of $A_{n}$ (since $G$ is simply decomposable, at most two components can fail to be subgroups of $A_{n}$ ); or only one component, namely, $C_{n, n+1}$, is not a subgroup of $A_{n}$ but $A_{n}$ is not a subgroup of $U_{n, n+1}$. For notational convenience we assume that one of these two possibilities first occurs when $n=0$

Suppose that when $A_{0}$ is deleted from the graph of $G$, two components, say $C_{0,1}$ and $C_{-1,0}$, arise which are not subgroups of $A_{0}$. Then

$$
G=C_{-1,0} *_{U_{-1,0}} A_{0} *_{U_{0,1}} C_{0,1}
$$

hence $G$ may be viewed as the tree product of $A_{0}$ and the vertices in $C_{-1,0}, C_{0,1}$. Let $A_{1}$ be the vertex of $C_{0,1}$ to which $A_{0}$ is joined by an edge. If $A_{1}$ is deleted from the vertices in the tree product (2), the subtree determined by the vertices of $C_{-1,0}$ and $A_{0}$ will be one component whose tree product is not a subgroup of $A_{1}$; hence there can be at most one other component, say $C_{1,2}$, not a subgroup of $A_{1}$. Hence

$$
G=C_{-1,0} *_{U_{-1,0}} A_{0} *_{U_{0,1}} A_{1} *_{U_{1,2}} C_{1,2} .
$$

Similarly, working with the left component $C_{-1,0}$ we obtain

$$
G=C_{-2,-1} *_{U_{-2,-1}} A_{-1} *_{U-1,0} A_{0} *_{U_{0,1}} A_{1} *_{U_{1,2}} C_{1,2}
$$

Continuing in this way, we can show that

$$
G=\cdots *_{U_{-3,-2}} A_{-2} *_{U_{-2,-1}} A_{-1} *_{U_{-1,0}} A_{0} *_{U_{0,1}} A_{1} *_{U_{1,2}} A_{2} *_{U_{2,3}} \cdots
$$

(where the sequence may have finitely many positive or negative indices); indeed, the vertices of $G$ which are not subgroups of

$$
\operatorname{gp}\left(A_{-n}, \ldots, A_{-1}, A_{0}, A_{1}, \ldots, A_{n}\right)
$$

have levels greater than $n$. 
Now, $C_{-1,0}=\operatorname{gp}\left(A_{-1}, A_{-2}, \ldots\right)$, and $C_{0,1}=\operatorname{gp}\left(A_{1}, A_{2}, \ldots\right)$; hence for some smallest positive integers $k, m$, we have $A_{-k} \nless A_{0}$ and $A_{m} \nless A_{0}$. Then

where

$$
G=C_{-k-1,-k} *_{V} A_{-k} *_{U} A_{0} *_{W} A_{m} *_{X} C_{m, m+1}
$$

$$
V=U_{-k-1,-k}, \quad U=U_{-k,-k+1}, \quad W=U_{m-1, m}, \quad \text { and } \quad X=U_{m, m+1} .
$$

Clearly, $A_{-k} \neq U$ and $A_{m} \neq W$. Writing $G$ as

$$
G=C_{-k,-k+1} *_{U} A_{0} *_{W} C_{m-1, m},
$$

we see that $U=A_{0}=W$. Hence

$$
\begin{aligned}
G & =C_{-k-1,-k} *_{V} A_{-k} *_{U} A_{m} *_{X} C_{m, m+1} \\
& =C_{-k,-k+1} *_{U} A_{m} *_{X} C_{m, m+1} \\
& =C_{-k-1,-k} *_{V} A_{-k} *_{U} C_{m-1, m} .
\end{aligned}
$$

Hence $C_{m, m+1}=X$ and $C_{-k-1,-k}=V$. Thus $G=A_{-k} *_{U} A_{m}$ where $U \neq A_{-k}, A_{m}$. Since $U=A_{0}=W$, it follows that the vertices $A_{j}$ for $-k<j<m$ are all equal to $U$.

This completes the argument for the case of two components.

Suppose next that only one component $C_{0,1}$ not a subgroup of $A_{0}$ arises when $A_{0}$ is deleted from the graph of $G$, and that $A_{0}$ is not a subgroup of $U_{0,1}$. This case is treated in a manner similar to the preceding one except that here one obtains

$$
G=A_{0} *_{U_{0,1}} A_{1} *_{U_{1,2}} A_{2} *_{U_{2,3}} \cdots
$$

which is infinite in at most one direction. Arguing as above, one shows that $G=A_{0} *_{U} A_{n}$ where $U=U_{n-1, n}$ and $n$ is the smallest positive integer $j$ such that $A_{j} \nless A_{0}$. It follows that all other vertices on the simple path joining $A_{0}$ to $A_{n}$ are equal to $U$.

Finally, suppose that $G$ has several vertices of level zero. Contract the subtree of vertices of level zero to a single vertex $L_{0}$. Then the above arguments show that either

$$
G=L_{0}
$$

$G$ is the ascending union of

$$
\begin{gathered}
L_{0}<A_{1}<A_{2}<\cdots, \\
G=\left(A_{i} * A_{j} ; U\right), \quad U \neq A_{i}, A_{j},
\end{gathered}
$$

or

$$
G=\left(A_{k} * L_{0} ; U\right), \quad U \neq A_{k}, L_{0} .
$$

In (3), redefine the levels of the vertices in $L_{0}$ so that there is a unique vertex of level zero. In (4), replace $L_{0}$ by its vertex $A_{0}$ which is joined by an edge to $A_{1}$. In 
(6), redefine levels of the vertices $A_{k}$ and those of $L_{0}$ so that $A_{k}$ is the unique vertex of level zero.

This completes the proof of Theorem 3.

THEOREM 4. Let $G$ be a tree product

$$
\Pi *\left(A_{i} ; U_{j k}=U_{k j}\right)
$$

and suppose each amalgamated subgroup $U_{j k}$ is finitely generated. If $G$ is finitely generated, then each factor $A_{i}$ is finitely generated and $G$ is the tree product of finitely many of its factors.

Proof. Since $G$ is finitely generated, $G$ is generated by finitely many $A_{i}$, say $A_{1}, \ldots, A_{n}$ which are the vertices of a subtree. Now this finite tree has an extremal vertex, say $A_{n}$, which is joined by an edge to exactly one vertex. Then the vertices $A_{1}, \ldots, A_{n-1}$ form a subtree with tree product $G_{n}$, and $G$ is the free product of $A_{n}$ and $G_{n}$ with a finitely generated subgroup amalgamated (by Theorem 1). It follows that $A_{n}$ and $G_{n}$ are finitely generated. For, if $(C * D ; V)$ is finitely generated and $V$ is finitely generated, but say $C$ were not finitely generated, then $C$ would be a proper ascending union of finitely generated subgroups $C_{j}$ each of which contains $V$; hence $(C * D ; V)$ would be the ascending union of $\left(C_{j} * D ; V\right)$, which is proper since $\left(C_{j} * D ; V\right) \cap C=C_{j}$.

Repetition of this argument shows that $A_{n-1}, \ldots, A_{1}$ are also finitely generated.

Since any factor $A_{k}$ could have been included in $A_{1}, \ldots, A_{n}$, each factor $A_{k}$ is finitely generated.

4. HNN groups. Two constructions enter our description of the subgroups of $G=(A * B ; U)$. The first, the tree product, has already been discussed. The second has been used by several authors: Higman, Neumann and Neumann [6] use the construction to establish certain embedding properties; Higman [5] uses it in his paper on subgroups of finitely presented groups; Moldavanski [10] uses it to obtain properties about groups with one defining relation; W. W. Boone has informed us that this construction is used in various proofs of the unsolvability of the word problem, see, in particular, J. L. Britton's paper on the word problem in Ann. of Math. (2) 77 (1963), 16-32, MR \#5891.

Definition. Let $K$ be a group and let $\left\{\varphi_{i}\right\}$ be a collection of isomorphisms of subgroups $L_{i}$ into $K$. Then we call the group

$$
G=\left\langle t_{1}, t_{2}, \ldots, K ; \text { rel } K, t_{1} L_{1} t_{1}^{-1}=\varphi_{1}\left(L_{1}\right), t_{2} L_{2} t_{2}^{-1}=\varphi_{2}\left(L_{2}\right), \ldots\right\rangle,
$$

the $H N N$ group with base $K$ and associated isomorphisms $\left\{\varphi_{i}\right\}$, and denote it by $\operatorname{HNN}\left(K,\left\{\varphi_{i}\right\}\right)$; the associated subgroups are $\left\{L_{i}, \varphi_{i}\left(L_{i}\right)\right\}$ and the free part of $G$ is the subgroup generated by $t_{1}, t_{2}, \ldots$.

It is easy to see that the free part of $G$ is a free group freely generated by $t_{1}, t_{2}, \ldots$, has trivial intersection with the normal subgroup of $G$ generated by $K$, and hence 
is a homomorphic image of $G$. (Conversely, if a group $G$ has a free group as a homomorphic image, $G$ is an HNN group whose base and associated subgroups all equal the kernel, and whose free part is a properly chosen representative system.)

A basic property of HNN groups is the following:

Let $\left\{x_{i}\right\},\left\{y_{i}\right\}$ be sets of the same cardinality as $\left\{\varphi_{i}\right\}$, and let $X, Y$ be the free groups on $\left\{x_{i}\right\}$ and on $\left\{y_{i}\right\}$, respectively. Then the free product $X * \operatorname{HNN}\left(K,\left\{\varphi_{i}\right\}\right)$ is isomorphic to the amalgamated product

$$
\left((X * K) *(Y * K) ; K * x_{1} L_{1} x_{1}^{-1} * \cdots=K * y_{1} \varphi_{1}\left(L_{1}\right) y_{1}^{-1} * \cdots\right)
$$

where $t_{i}$ is mapped onto $y_{i}^{-1} x_{i}$, and $X, K$ are mapped identically onto themselves.

It follows that $K$ is a subgroup of $\operatorname{HNN}(K,\{\varphi\})$ in the natural way. Hence, $\operatorname{HNN}\left(K,\left\{\varphi_{i}\right\}\right)$ with nontrivial free part is decomposable (as an amalgamated product) in a nontrivial manner unless its free part is cyclic and its base $K$ is generated by its associated subgroups $\left\{L_{i}, \varphi_{i}\left(L_{i}\right)\right\}$; indeed

$$
\operatorname{HNN}\left(K,\left\{\varphi_{i}\right\}\right)=\Pi_{i}^{*}\left(\operatorname{HNN}\left(K, \varphi_{i}\right) ; K\right)=K_{K^{\prime}}^{*} \operatorname{HNN}\left(K^{\prime},\left\{\varphi_{i}\right\}\right)
$$

where $K^{\prime}$ is the subgroup of $K$ generated by $\left\{L_{i}, \varphi_{i}\left(L_{i}\right)\right\}$. (In particular, any group having a noncyclic free group as a homomorphic image is nontrivially decomposable as an amalgamated product.) Moreover, if $K>M>K^{\prime}$, then $\mathrm{gp}\left(t_{1}, t_{2}, \ldots, M\right)$ equals $\operatorname{HNN}\left(M,\left\{\varphi_{i}\right\}\right)$ and intersects $K$ in $M$; for,

$$
\operatorname{HNN}\left(K,\left\{\varphi_{i}\right\}\right)=K_{M}^{*} \operatorname{HNN}\left(M,\left\{\varphi_{i}\right\}\right) .
$$

LemMA 2. Let $G=H N N\left(K,\left\{\varphi_{i}\right\}\right)$ and let $t_{1}, t_{2}, \ldots$ freely generate the free part of $G$. Then the normal subgroup $N$ of $G$ generated by $K$ is a tree product whose vertices correspond to the conjugates of $K$ by the freely reduced words in the $t_{i}$; an edge joins $W t_{i}^{\varepsilon} K t_{i}^{-\varepsilon} W^{-1}$ to $W K W^{-1}$, and this edge corresponds to the subgroup $W t_{i}^{\varepsilon} L_{i} t_{i}^{-\varepsilon} W^{-1}=W \varphi_{i}\left(L_{i}\right) W^{-1}$ if $\varepsilon=1$, and to $W t_{i}^{\varepsilon} \varphi_{i}\left(L_{i}\right) t_{i}^{-\varepsilon} W^{-1}=W L_{i} W^{-1}$ if $\varepsilon=-1$.

Proof. Using the Reidemeister-Schreier method with the freely reduced words in $t_{i}$ as a Schreier representative system for $G \bmod N$, we have that $N$ is a partial generalized free product with the vertices and edges as specified. To show that $N$ is a tree product, define the level of a vertex $W K W^{-1}$ to be the word length of $W$. Then the conditions of Lemma 1 are satisfied.

Note that in the special case when the free part of $G$ is infinite cyclic, then the tree product $N$ reduces to a stem (path) infinite in both directions. This type of tree product arises in the study of groups with one defining relation and knot groups.

A simple consequence of Lemma 2 and the above is that the center of $\operatorname{HNN}\left(K,\left\{\varphi_{i}\right\}\right)$ whose free part is noncyclic is the center of $K$ intersected with the fixed sets of each of the $\varphi_{i}$. For, a central element must be in $K$ and each $L_{i}$, since the center of a nontrivial amalgamated product is in its amalgamated subgroup. 
Lemma 3. Let $H=H N N\left(K,\left\{\varphi_{i}\right\}\right)$ be finitely generated. Then the number of $\varphi_{i}$ is finite. If each of the associated subgroups $L_{i}$ is finitely generated, then $K$ is finitely generated. If $K$ is a tree product and each of the associated subgroups $L_{i}$ and $\varphi_{i}\left(L_{i}\right)$ is a subgroup of a vertex of $K$, then $K$ is the tree product of finitely many of its vertices.

Proof. The free part of $H$ is a homomorphic image of $H$ whose rank is the number of $\varphi_{i}$, and so this number must be finite.

Let $K^{\prime}$ be the subgroup of $K$ generated by the associated subgroups $\left\{L_{i}, \varphi_{i}\left(L_{i}\right)\right\}$.

Suppose each $L_{i}$ is finitely generated. If $K$ were not finitely generated, then $K$ would be a proper ascending union of finitely generated subgroups $K_{j}$ each of which contains $K^{\prime}$. Hence $H$ would be the ascending union of subgroups $H_{j}$ $=\mathrm{HNN}\left(K_{j},\left\{\varphi_{i}\right\}\right)$; this chain is proper (since $H_{j} \cap K=K_{j}$ ), contrary to $H$ being finitely generated. Thus $K$ is finitely generated if the $L_{i}$ are.

To prove the last assertion, suppose $K$ were a tree product not generated by finitely many of its vertices. Clearly, since $H$ is finitely generated, $K$ is generated by countably many of its vertices. Hence $K$ is a proper ascending union of subgroups $K_{j}$ where $K_{j}$ is a tree product of finitely many vertices of $K$ including the finitely many which have $\left\{L_{i}, \varphi_{i}\left(L_{i}\right)\right\}$ as subgroups. Then $K_{j}>K^{\prime}$ and $H$ would be the proper ascending union of $\operatorname{HNN}\left(K_{j},\left\{\varphi_{i}\right\}\right)$, contrary to $H$ being finitely generated.

5. Compatible regular extended Schreier systems. Our proof of the subgroup theorem makes use of a specially chosen coset representative system which is a generalization of a regular extended Schreier system as defined in [9, p. 239].

Let $G$ be a group generated by two of its subgroups $A, B$ whose intersection is $U$. Suppose $u_{1}, u_{2}, \ldots$ are generating symbols for $U ; a_{1}, a_{2}, \ldots, u_{1}, u_{2}, \ldots$ are generating symbols for $A$; and $b_{1}, b_{2}, \ldots, v_{1}, v_{2}, \ldots$ are generating symbols for $B$ where $v_{i}$ defines the same element of $U$ as $u_{i}$. We call $a_{1}, a_{2}, \ldots a$-generators; $b$-, $u$-, $v$-generators are defined similarly; also $a$ - or $u$-generators are called $\alpha$ generators; $b$ - or $v$-generators, $\beta$-generators. An $x$-symbol means an $x$-generator or its inverse where $x$ is $a, b, u, v, \alpha$ or $\beta$.

Let $H$ be a subgroup of $G$. By a compatible regular extended Schreier system (cress) for $G \bmod H$ (on the given generators for $G$ ), we mean a pair of right coset representative functions for $G \bmod H$, called the $\alpha$ - and $\beta$-representative functions respectively, with the following properties:

(1) The representative functions form a regular extended Schreier system for $G \bmod H$ (where we choose the $\alpha$-type representatives to be the neutral representatives), i.e.,

(1.1) if a representative $N=M z$ (where $z$ is an $\alpha$ - or $\beta$-symbol), then $N$ and $M$ are both representatives of the same type ( $\alpha$ - or $\beta$-) as the symbol $z$;

(1.2) if the $\alpha$-symbols are deleted completely from the ends of the $\alpha$-representatives, the resulting words form a double coset representative system for $G \bmod (H, A)$ 
which we call the associated $\alpha$-double coset representative system, and analogous demands are made for the $\beta$-representatives.

(2) When the $u$-symbols ( $v$-symbols) are deleted completely from the ends of the $\alpha$-representatives ( $\beta$-representatives), the resulting words form a double coset representative system for $G \bmod (H, U)$ called the associated $u$-double coset (v-double coset) representative system.

(3) An $\alpha$-representative does not end in a $v$-symbol, and a $\beta$-representative does not end in a $u$-symbol.

(4) If $K$ is both a $u$ - and a $v$-double coset representative, then $K P\left(u_{i}\right)$ is an $\alpha$-representative iff $K P\left(v_{i}\right)$ is a $\beta$-representative (where $P\left(u_{i}\right)$ is a word in the $u$ generators and $P\left(v_{i}\right)$ is the corresponding word in the $v$-generators).

Lemmas 4 and 5 are used in constructing a cress for $G \bmod H$.

Lemma 4. Let $\left\{E_{i}\right\}$ be a double coset representative system for a group $C \bmod a$ pair of subgroups $(J, U)$. Then a collection of words $\left\{E_{i} P_{i j}\right\}$ where $P_{i j}$ is in $U$ is a coset representative system for $C \bmod J$ iff $\left\{P_{i j}\right\}$ is a coset representative system for $U \bmod U \cap E_{i}^{-1} J E_{i}$. More generally, if $N$ is a subgroup of $U$, then the collection $\left\{E_{i} P_{i j}\right\}$ is a double coset representative system for $C \bmod (J, N)$ iff $\left\{P_{i j}\right\}$ is a double coset representative system for $U \bmod \left(U \cap E_{i}^{-1} J E_{i}, N\right)$.

Lemma 5. Let $C$ be a group with generators $c_{1}, c_{2}, \ldots, u_{1}, u_{2}, \ldots$, let $U=$ $\mathrm{gp}\left(u_{1}, u_{2}, \ldots\right)$, and let $J$ be any subgroup of $C$. Then there exists a Schreier system for $C \bmod J$ such that when the u-symbols are completely deleted from the ends of the representatives, the resulting words form a double coset representative system for $C \bmod (J, U)$.

Proof. Define the length of a double coset in $C \bmod (J, U)$ as the minimum length of a word contained in the double coset.

We construct our Shreier system inductively, selecting double coset representatives $E_{i}$ for $C \bmod (J, U)$, and then supplementing $E_{i}$ with any Schreier system for $U \bmod U \cap E_{i}^{-1} J E_{i}$.

For the double coset $J U$ of length zero, we select 1 as its double coset representative. Assume we have selected representatives for the cosets of $J$ contained within the double cosets of $(J, U)$ of length less than $r$; and let $J W U$ be a double coset of length $r>0$ with $W$ of length $r$; then $W$ does not end in a $u$-symbol. If $W=Q x$ where $x$ is a $c$-symbol, then $Q$ is in a double coset $J Q U$ of length less than $r$; hence the representative $\bar{Q}$ of $Q$ has been selected: set the double coset representative $E_{i}$ of $J W U$ to be the word $\bar{Q} x$, which clearly does not end in a $u$-symbol.

We refer to a Schreier representative system of the kind described in Lemma 5 as a special Schreier system for $C \bmod J$ with respect to $U$.

Lemma 6. Let $G, A, B, U, H$ be as described at the beginning of this section. Then there exists a cress for $G \bmod H$ on the given generators.

Proof. Given two representative systems for $G \bmod H$, called $\alpha$ - and $\beta$-repre- 
sentative systems, express each $\alpha$-representative ${ }^{\alpha} W$ as a product of three words ${ }^{\alpha} W=D_{\alpha} E_{u} P$ where $P$ is the maximal terminal segment of ${ }^{\alpha} W$ containing only $u$-symbols and $E_{u} P$ is the maximal terminal segment containing only $\alpha$-symbols; similarly, express each $\beta$-representative as a product of three words $D_{\beta} E_{v} Q$.

It is easy to show that the following conditions (5)-(9) are equivalent to conditions (1)-(3) in the definition of a cress:

(5) The collection $\left\{D_{\alpha}\right\}$ is a double coset representative system for $G \bmod (H, A)$ and each $D_{\alpha}$ is a $\beta$-representative ending in a $b$-symbol (unless $D_{\alpha}=1$ ); an analogous statement for the collection $\left\{D_{\beta}\right\}$.

(6) The collection $\left\{D_{\alpha} E_{u}\right\}$ is a double coset representative system for $G \bmod (H, U)$; an analogous statement for the collection $\left\{D_{\beta} E_{v}\right\}$.

(7) For a fixed $D_{\alpha}$, the collection $\left\{E_{u} P\right\}$ of words which go with this $D_{\alpha}$ is a Schreier system for $A \bmod A \cap D_{\alpha}^{-1} H D_{\alpha}$; an analogous statement for a fixed $D_{\beta}$.

(8) For a fixed $D_{\alpha}$, the associated collection $\left\{E_{u}\right\}$ is a double coset representative system for $A$ mod $\left(A \cap D_{\alpha}^{-1} H D_{\alpha}, U\right)$; an analogous statement for a fixed $D_{\beta}$.

(9) For a fixed $D_{\alpha} E_{u}$, the collection $\{P\}$ of words which go with this $D_{\alpha} E_{u}$ is a Schreier system for $U \bmod U \cap E_{u}^{-1} D_{\alpha}^{-1} H D_{\alpha} E_{u}$; an analogous statement for a fixed $D_{\beta} E_{v}$.

Conditions (7), (8), and (9) merely state that for a fixed $D_{\alpha}$ the associated collection $\left\{E_{u} P\right\}$ is a special Schreier system for $A \bmod A \cap D_{\alpha}^{-1} H D_{\alpha}$ with respect to $U$ and analogously for fixed $D_{\beta}$.

We construct a cress by an inductive procedure similar to that used in constructing a regular extended Schreier system (see, for example, [9, p. 241]). By the syllable length of a double coset of $(H, A)$ or of $(H, B)$ we mean the minimum syllable length (with respect to $\alpha$ - and $\beta$-syllables) of a word in the double coset.

The inductive procedure consists in selecting $\alpha$-representatives (and $\beta$-representatives) for all cosets of $H$ within those double cosets $H W A$ (and $H W B$ ) of length $n$; to do this we first select double coset representatives of minimum syllable length and then supplement these with a special Schreier system.

For $n=0$, choose 1 as both the $\alpha$ - and $\beta$-double coset representatives of $H A$ and $H B$ respectively. To obtain the $\alpha$-representatives for the cosets of $H$ in $H A$ we supplement the $\alpha$-double coset representative 1 with a special Schreier system for $A$ mod $A \cap H$ (which is $A \cap 1^{-1} H 1$ ) with respect to $U$. Similarly for $\beta$-representatives for cosets of $H$ in $H B$.

Assume we have selected $\alpha$-representatives ( $\beta$-representatives) for all cosets of $H$ contained in a double coset of $(H, A)$ (of $(H, B))$ of syllable length less than $r$. Let $H W A$ and $W$ have syllable length $r>0$. Now $W$ ends in a $\beta$-symbol; hence the double coset $H W B$ has syllable length less than $r$. Thus ${ }^{\beta} W$ has been selected and ${ }^{\beta} W$ can be written ${ }^{\beta} W=D_{\beta} E_{v} Q$; choose $D_{\beta} E_{v}$ as the $\alpha$-double coset representative $D_{\alpha}$ of $H W A$. Since the syllable length of $D_{\beta} \leqq r-1$ while the syllable length of $D_{\beta} E_{v} \geqq r$, we must have $E_{v} \neq 1$ and so $E_{v}$ ends in a $b$-symbol. We then supplement $D_{\alpha}$ with a special Schreier system for $A \bmod A \cap D_{\alpha}^{-1} H D_{\alpha}$ with respect to $U$. 
In analogous fashion select $\beta$-representatives for all cosets of $H$ within a double coset of $(H, B)$ of syllable length $r$.

From the construction of the $\alpha$ - and $\beta$-representative systems it is clear that conditions (5)-(9) and hence (1)-(3) are satisfied. To satisfy condition (4), note that if $K$ is both a $u$ - and a $v$-double coset representative, then in the course of the above construction, $K$ was supplemented by a Schreier system in $u$-generators and one in $v$-generators for $U \bmod U \cap K^{-1} H K$; once one of these Schreier systems has been chosen, select the second one to be the corresponding set of words obtained by interchanging $u_{i}$ and $v_{i}$.

Corollary. Let $G, A, B, U, H$ be as above. Suppose $K$ is a $u$-or v-double coset representative in a cress for $G \bmod H$ on the given generators. If $K=K_{1} K_{2} \cdots K_{n}$ is the factorization of $K$ into $\alpha$-and $\beta$-syllables, then no $K_{i}$ defines an element of $U$. In particular, if $G=(A * B ; U)$, then the syllable length of $K$ and the length of the element defined by $K$ in $G$ coincide.

Proof. It is clear from the definition of a cress than an $\alpha$-or $\beta$-double coset representative is both a $u$ - and a $v$-double coset representative; hence if the last syllable ( $\alpha$ - or $\beta$-) of any representative is deleted, then the resulting word is both a $u$ - and a $v$-double coset representative. It therefore suffices to show that the last syllable $K_{n}$ of $K$ is not in $U$. Suppose $K_{n}$ were in $U$. Since $K_{1} \cdots K_{n-1}$ is both a $u$ - and a $v$-double coset representative, $K_{1} \cdots K_{n-1}=K$, contrary to $K_{n}$ being the last syllable of $K$ and hence nonempty.

LEMMA 7. Let $G$ and $H$ be as above, and let $\tau$ be a Kuroš rewriting process using $a$ cress for $G \bmod H$. Then in the presentation obtained for $H$ using $\tau$, the following hold:

(10) the subgroup of $H$ generated by the symbols $s_{K, x}$ where $K$ ranges over the $\alpha$-representative with a fixed double coset representative $D_{\alpha}$ and $x$ ranges over all a-generators is $D_{\alpha} A D_{\alpha}^{-1} \cap H=A_{H^{\alpha}}^{D^{\alpha}}$;

(11) the subgroup of $H$ generated by the symbols $s_{K, u_{i}}$ where $K$ ranges over the $\alpha$-representatives with a fixed $u$-double coset representative $D_{\alpha} E_{u}$ and $u_{i}$ ranges over all u-generators is $U_{H^{\alpha}}^{D E_{u}}$.

Assertions analogous to (10) and (11) hold for s-generators associated with $\beta$ representatives, $\beta$-generators and v-generators.

(12) $H$ is itself generated by the s-generators and the t-generators $t_{L}$ where $L$ ranges over the $v$-double coset representatives.

Proof. (10) follows from Lemma 4.8 of [9, p. 240].

(11) follows from the fact that the $\alpha$-representative of $D_{\alpha} E_{u} P u_{i}$ is

where

$$
D_{\alpha} E_{u} \overline{P u_{i}}
$$

$$
\overline{P u_{i}}
$$

is the Schreier representative of $P u_{i}$ in $U \bmod U \cap E_{u}^{-1} D_{\alpha}^{-1} H D_{\alpha} E_{u}$. 
We know that $H$ is generated by all the $s$-generators and all the $t$-generators. We now wish to show that we may restrict the $t$-generators to those associated with $v$-double coset representatives. Since the neutral representatives are the $\alpha$-representatives, we need only the $t$-generators associated with $\beta$-representatives. We show that $t_{D_{\beta} E_{v} Q(v)}$ can be solved for in terms of $t_{D_{\beta} E_{v}}$ and $s$-generators of the form $s_{D_{\alpha} E_{u} P, u_{i}}$ where $D_{\alpha} E_{u}$ is the $u$-double coset representative of $D_{\beta} E_{v}$. Specifically suppose that ${ }^{\alpha}\left(D_{\beta} E_{v}\right)=D_{\alpha} E_{u} M$. Then we show that

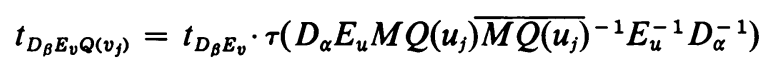

where

$$
\overline{M Q\left(u_{j}\right)}
$$

is the Schreier representative of $M Q\left(u_{j}\right)$ in $U \bmod U \cap E_{u}^{-1} D_{\alpha}^{-1} H D_{\alpha} E_{u}$.

To show that the LHS and RHS of (13) define the same element of $H$, note that $t_{D_{\beta} E_{v} Q\left(v_{j}\right)}$ defines the element

$$
\begin{aligned}
D_{\beta} E_{v} Q\left(v_{j}\right)^{\alpha}\left(D_{\beta} E_{v} Q\left(v_{j}\right)\right)^{-1} & =D_{\beta} E_{v} Q\left(u_{j}\right)^{\alpha}\left(D_{\alpha} E_{u} M Q\left(u_{j}\right)\right)^{-1} \\
& =D_{\beta} E_{v} Q\left(u_{j}\right)\left(D_{\alpha} E_{u} \overline{M Q\left(u_{j}\right)}\right)^{-1} \\
& =D_{\beta} E_{v}\left(D_{\alpha} E_{u} M\right)^{-1} D_{\alpha} E_{u} M Q\left(u_{j}\right) \overline{M Q\left(u_{j}\right)}-1
\end{aligned}
$$

which defines the same element as

$$
t_{D_{\beta} E_{v}} \cdot \tau\left(D_{\alpha} E_{u} M Q\left(u_{j}\right) \overline{M Q\left(u_{j}\right)^{-1}} E_{u}^{-1} D_{\alpha}^{-1}\right)
$$

6. The subgroup theorem. We shall prove that every subgroup $H$ of

$$
G=(A * B ; U)
$$

is an HNN group of the form

$$
H=\left\langle t_{1}, t_{2}, \ldots, S ; \text { rel } S, t_{1} U_{H}^{\delta_{1}} t_{1}^{-1}=U_{H}^{\delta_{1}}, t_{2} U_{H}^{\delta_{2}} t_{2}^{-1}=U_{H}^{\delta_{2}}, \ldots\right\rangle
$$

where $S$ is a tree product whose vertices are conjugates of $A$ or $B$ intersected with $H$, and whose amalgamated subgroups are conjugates of $U$ intersected with $H$. Thus $H$ is an HNN group (with possibly trivial free part) whose base is $S$ and whose associated subgroups are conjugates of $U$ intersected with $H$. Moreover, each of these associated subgroups is contained in a vertex of the tree product $S$.

TheOREM 5. Let $G=(A * B ; U)$ and let $H$ be a subgroup of $G$. Suppose $\left\{D_{\alpha} E_{u} P\right\}$ and $\left\{D_{\beta} E_{v} Q\right\}$ are the $\alpha$ - and $\beta$-representatives of a cress for $G \bmod H$. Then $H$ is generated by those $t_{D_{\beta} E_{v}}$ such that $D_{\beta} E_{v}$ is neither an $\alpha$-nor $\beta$-double coset representative together with all the subgroups $A_{H^{\alpha}}^{D^{\alpha}}$ and $B_{H^{\beta}}^{D}$. Moreover, the following hold:

(1) The $t_{D_{\beta} E_{v}}$ such that $D_{\beta} E_{v}$ is not an $\alpha$-or $\beta$-double coset representative freely generate a free group $F$. 
(2) All the subgroups $A_{H^{\alpha}}^{D^{\alpha}}$ and $B_{H^{\beta}}^{D}$ generate a tree product $S$ in which $A_{H^{\alpha}}^{D^{\alpha}}$ and $B_{H^{\beta}}^{D_{\beta}}$ are neighboring vertices whenever $D_{\alpha}=D_{\beta}=1$, or $D_{\alpha}$ and $D_{\beta}$ are " a syllable apart", i.e., $D_{\alpha}$ is obtained by deleting the last syllable from $D_{\beta}$ or vice versa; if $A_{H^{\alpha}}^{D^{\alpha}}$ and $B_{H^{\beta}}^{D^{\beta}}$ are neighbors and $D$ is the longer of $D_{\alpha}, D_{\beta}$, then $U_{H}^{D}$ is the subgroup amalgamated between them under the identity mapping.

(3) $H$ is the HNN group

$$
\left\langle\ldots, t_{D_{\beta} E_{v}}, \ldots, S ; \text { rel } S, \ldots, t_{D_{\beta} E_{v}} U_{H^{\alpha}}^{D^{\alpha} E_{u}} t_{D_{\beta} E_{v}}^{-1}=U_{H^{\beta}}^{D_{v} E_{v}}, \ldots\right\rangle
$$

where $D_{\beta} E_{v}$ is as in (1) and $D_{\alpha} E_{u}$ is the u-double coset representative of $D_{\beta} E_{v}$.

Proof. Let $G$ be presented by

$$
\begin{array}{r}
\left\langle a_{1}, a_{2}, \ldots, u_{1}, u_{2}, \ldots, b_{1}, b_{2}, \ldots, v_{1}, v_{2}, \ldots ; R_{11}\left(a_{i}, u_{j}\right), R_{12}\left(a_{i}, u_{j}\right) \ldots\right. \\
\left.R_{21}\left(b_{i}, v_{j}\right), R_{22}\left(b_{i}, v_{j}\right), \ldots, u_{1}=v_{1}, u_{2}=v_{2}, \ldots\right\rangle
\end{array}
$$

where

$$
\begin{aligned}
& A=\left\langle a_{1}, a_{2}, \ldots, u_{1}, u_{2}, \ldots ; R_{11}\left(a_{i}, u_{j}\right), R_{12}\left(a_{i}, u_{j}\right), \ldots\right\rangle, \\
& B=\left\langle b_{1}, b_{2}, \ldots, v_{1}, v_{2}, \ldots ; R_{21}\left(b_{i}, v_{j}\right), R_{22}\left(b_{i}, v_{j}\right), \ldots\right\rangle,
\end{aligned}
$$

and $U=\mathrm{gp}\left(u_{1}, u_{2}, \ldots\right)=\mathrm{gp}\left(v_{1}, v_{2}, \ldots\right)$. Let $\tau$ be the Kuroš rewriting process using the given cress for $G \bmod H$.

By Theorem 4.8 of [9], $H$ has generators $\left\{s_{N, x}\right\}$ and $\left\{t_{N}\right\}$ where $N$ ranges over the $\alpha$ - and $\beta$-representatives and $x$ ranges over the generators of the same type ( $\alpha$ - or $\beta$-) as the representative $N$; and $H$ has as defining relators

(4) $S_{N, x}$ whenever $N x$ is freely equal to a representative,

(5) $t_{N}$ if $N$ is an $\alpha$-representative,

(6) $\tau\left(N R_{1 n} N^{-1}\right)$ where $N$ ranges over the $\alpha$-representatives,

(7) $\tau\left(N R_{2 n} N^{-1}\right)$ where $N$ ranges over the $\beta$-representatives, and

(8) $\tau\left(N u_{i} v_{i}^{-1} N^{-1}\right)$ where $N$ ranges over the $\beta$-representatives.

A relator in (6) simplifies to a word in $s$-generators alone each of the form $s_{\alpha(N W), x}$ where $W$ is an initial segment of $R_{1 n}$ and $x$ is an $\alpha$-generator; the $\alpha$-double coset representative of ${ }^{\alpha}(N W)$ is the same as that of $N$. Thus if we collect those relators in (6) according to the $\alpha$-double coset representative $D_{\alpha}$ of $N$, we obtain the relators $\tau\left(D_{\alpha} \cdot E_{u} P R_{1 n} P^{-1} E_{u}^{-1} D_{\alpha}^{-1}\right)$ where $\left\{E_{u} P\right\}$ is a Schreier system for $A \bmod A \cap D_{\alpha}^{-1} H D_{\alpha}$. Now if $\tau^{\prime}$ is the Reidemeister-Schreier rewriting process associated with the Schreier system $\left\{E_{u} P\right\}$ for $A \bmod A \cap D_{\alpha}^{-1} H D_{\alpha}$, it is clear that the $s_{E_{u} P, x}$ such that

$$
\overline{E_{u} P x} \approx E_{u} P x
$$

together with $\tau^{\prime}\left(E_{u} P R_{1 n} P^{-1} E_{u}^{-1}\right)$ form a set of defining relators for $A \cap D_{\alpha}^{-1} H D_{\alpha}$ on the generators $s_{E_{u} P, x}$. The element in $H$ defined by $s_{D_{\alpha} E_{u} P, x}$ is just the conjugate by $D_{\alpha}$ of the element in $A \cap D_{\alpha}^{-1} H D_{\alpha}$ defined by $s_{E_{u} P, x}$. Hence, those $s_{N, x}$ where $x$ is any $\alpha$-generator and $N$ has $\alpha$-double coset representative $D_{\alpha}$ together with the relators in (4) and (6) involving such $N$ give a presentation for $A_{H}^{D^{\alpha}}$. Analogous 
remarks with regard to the relators in (4) and (7) show that (4), (6) and (7) can be partitioned into sets of defining relators for the groups $A_{H^{\alpha}}^{D^{\alpha}}$ and $B_{H^{\beta}}^{D^{\beta}}$.

Next we consider the relators in (8). If ${ }^{\alpha} N=D_{\alpha} E_{u} P$ and $N=D_{\beta} E_{v} Q$, then (8) reduces to

$$
s_{D_{\beta} E_{v} Q, v_{\iota}}=t_{D_{\beta} E_{v} Q} S_{D_{\alpha} E_{u} P, u_{\iota}} t_{D_{\beta} E_{v} \overline{Q v} v_{i}}^{-1}
$$

where $\overline{Q v_{i}}$ is the Schreier representative of $Q v_{i}$ in $U \bmod U \cap\left(D_{\beta} E_{v}\right)^{-1} H D_{\beta} E_{v}$. From the relators (4)-(8) one can derive the formula (see (13) in the proof of Lemma 7)

$$
t_{D_{\beta} E_{v} Q\left(v_{f}\right)}=t_{D_{\beta} E_{v}} \cdot \tau\left(D_{\alpha} E_{u} M Q\left(u_{j}\right) \overline{M Q\left(u_{j}\right)}-1 E_{u}^{-1} D_{\alpha}^{-1}\right)
$$

where ${ }^{\alpha}\left(D_{\beta} E_{v}\right)=D_{\alpha} E_{u} M$. (Clearly, $P=\overline{M Q\left(u_{j}\right)}$.) Using (10), (9) can be replaced by

$$
\begin{aligned}
s_{D_{\beta} E_{v} Q, v_{i}}= & \left.t_{D_{\beta} E_{v}} \cdot \tau\left(D_{\alpha} E_{u} M Q\left(u_{j}\right) \overline{M Q\left(u_{j}\right.}\right)^{-1} E_{u}^{-1} D_{\alpha}^{-1}\right) \cdot \tau\left(D_{\alpha} E_{u} P u_{i} \overline{P u_{i}^{-1}} E_{u}^{-1} D_{\alpha}^{-1}\right) \\
(11) \quad & \cdot \tau\left(D_{\alpha} E_{u} \overline{M \overline{Q\left(u_{j}\right) u_{i}}} \overline{\left.Q\left(u_{j}\right) u_{i}^{-1} M^{-1} E_{u}^{-1} D_{\alpha}^{-1}\right) \cdot t_{D_{\beta} E_{v}}^{-1}}\right. \\
= & t_{D_{\beta} E_{v}} \cdot \tau\left(D_{\alpha} E_{u} M Q\left(u_{j}\right) u_{i} \overline{M Q\left(u_{j}\right) u_{i}^{-1}} \overline{M \overline{Q\left(u_{j}\right) u_{i}}} \overline{\left.Q\left(u_{j}\right) u_{i}^{-1} M^{-1} E_{u}^{-1} D_{\alpha}^{-1}\right) t_{D_{\beta} E_{v}}^{-1}}\right.
\end{aligned}
$$

which has the form

$$
t_{D_{\beta} E_{v}} W\left(s_{D_{\alpha} E_{u} L, u_{j}}\right) t_{D_{\beta} E_{v}}^{-1}=s_{D_{\beta} E_{v} Q, v_{i}} .
$$

Thus by a Tietze transformation we may eliminate the generators $t_{D_{\beta} E_{v} Q(v)}$ where $Q\left(v_{f}\right) \neq 1$, provided we replace the relations (9) by (12); note that the $t$-generators we are eliminating do not occur in (5) since an $\alpha$-representative cannot end in a $v$-symbol. Consequently, the only defining relations of $H$ that now involve $t$ generators are those in (5) and (12).

Hence, when $H$ is divided by the normal subgroup generated by all the $s$ generators, the resulting group is freely generated by those $t_{D_{\beta} E_{v}}$ such that $D_{\beta} E_{v}$ is not an $\alpha$-representative. Now $D_{\beta} E_{v}$ is an $\alpha$-representative iff $D_{\beta} E_{v}$ is either an $\alpha$ - or $\beta$-double coset representative; indeed, if $E_{v}=1$ then $D_{\beta} E_{v}$ is an $\alpha$-double coset representative, and if $E_{v} \neq 1$ then $D_{\beta} E_{v}$ is an $\alpha$-representative ending in a $\beta$ generator and hence is an $\alpha$-double coset representative. This proves assertion (1).

In order to show (2), we first show that the $s$-generators in our presentation for $H$ together with those defining relations which involve only $s$-generators, namely (4), (6), (7) and those relations in (12) for which $t_{D_{B} E_{v}^{\prime}}=1$, present a group $S^{\prime \prime}$ which is the tree product described in (2); we show subsequently that this group $S^{\prime \prime}$ is a subgroup of $H$ in the natural way, i.e., $S^{n}=S$.

As we have already observed, the defining relators that enter our presentation for $S^{\prime \prime}$ from (4), (6) and (7) can be partitioned to yield sets of defining relators for the groups $A_{H^{\alpha}}^{D^{\alpha}}$ and $B_{H^{\beta}}^{D^{\beta}}$. Moreover, the defining relations that enter from (12) simplify to

$$
S_{D_{\alpha} E_{u} Q(u), u_{i}}=S_{D_{\beta} E_{v} Q\left(v_{j}\right), v_{i}} .
$$


For, $t_{D_{\beta} E_{v}}=1$ iff $D_{\beta} E_{v}=D_{\alpha} E_{u}$, and also $E_{v}$ or $E_{u}$ is 1 ; hence in (11), $M=1$ and (13) follows from condition (4) of the definition of a cress. If $D_{\beta} E_{v}$ is fixed, then the right-hand sides of (13) generate the subgroup $U_{H^{\beta}}^{D E_{v}}$ of $B_{H^{\beta}}^{D^{\beta}}$ while the left-hand sides generate the (same) subgroup $U_{H^{\alpha}}^{D^{\alpha} E_{u}}$ of $A_{H^{\alpha}}^{D^{\alpha}}$; the pairing in (13) is an isomorphism (the identity mapping) between these subgroups. Moreover, $A_{H^{\alpha}}^{D^{\alpha}}$ and $B_{H^{\beta}}^{D^{\beta}}$ are connected by relations in (13) iff $D_{\alpha} E_{u}=D_{\beta} E_{v}$ for some $E_{u}, E_{v}$ iff $E_{u}=1$ and $D_{\alpha}=D_{\beta} E_{v}$, or $E_{v}=1$ and $D_{\beta}=D_{\alpha} E_{u}$ iff $D_{\alpha}=D_{\beta}=1$ or $D_{\alpha}$ and $D_{\beta}$ are a syllable apart; hence, if $A_{H^{\alpha}}^{D^{\alpha}}$ and $B_{H^{\beta}}^{D^{\beta}}$ are connected by relations in (13), $E_{u}$ and $E_{v}$ are uniquely determined. It follows that $S^{\prime \prime}$ is a partial generalized free product of the groups $A_{H^{\alpha}}^{D^{\alpha}}$ and $B_{H^{\beta}}^{D^{\beta}}$.

In order to show that the graph of $S^{\prime \prime}$ is a tree, we use Lemma 1. Define the level of a vertex $A_{H^{\alpha}}^{D^{\alpha}}$ (or $B_{H^{\beta}}^{D^{\beta}}$ ) to be the syllable length of $D_{\alpha}$ (or $D_{\beta}$ ). The vertices of level zero are $A \cap H$ and $B \cap H$ and these are joined by an edge corresponding to $U \cap H$. Moreover, condition (2) of Lemma 1 is clearly satisfied. Therefore $S^{\prime \prime}$ is the tree product as described in (2).

To show (3), let $t_{D_{\beta} E_{v}} \neq 1$, and consider those defining relations in (12) which involve $t_{D_{\beta} E_{v}}$. We must show that the mapping

$$
s_{D_{\beta} E_{v} Q, v_{i}} \rightarrow W\left(s_{D_{\alpha} E_{u} L, u_{j}}\right)
$$

of an $s$-generator in the right-hand side of (12) into the word in $s$-generators on the left-hand side of (12) is an isomorphism between two subgroups of $S^{\prime \prime}$. Now the $s$-generators on the left-hand sides of (14) generate the subgroup $U_{H^{B}}^{D^{B}} E_{v}$ in $B_{H^{\beta}}^{D^{\beta}}$; therefore the words in the $s$-generators on the right-hand sides of (14) generate the subgroup

$$
t_{D_{\beta} E_{v}}^{-1} U_{H^{\beta}}^{D^{E}} t_{D_{\beta} E_{v}}=U_{H^{\alpha} E_{u}}^{E_{1}}
$$

in $H$ and therefore also in $A_{H^{\alpha}}^{D^{\alpha}}$. Moreover, the mapping in (14) is just the isomorphism between $U_{H^{\beta}}^{D E_{v}}$ and $U_{H^{\alpha}}^{D^{\alpha}} E_{u}$ induced by conjugation by $t_{D_{\beta} E_{v}}^{-1}$ in $H$. Since $A_{H^{\alpha}}^{D^{\alpha}}$ and $B_{H^{B}}^{D^{\beta}}$ are subgroups of their tree product $S^{\prime \prime}$ in the natural way, it follows that (14) is an isomorphism between the subgroups $U_{H^{B}}^{D^{\beta} E_{v}}$ and $U_{H^{\alpha}}^{D E_{u}}$ of $S^{\prime \prime}$. Hence $H$ is an HNN group with base $S^{\prime \prime}$, and so $S=S^{\prime \prime}$. Consequently, (2) and (3) follow and the theorem is proved.

COROLlARY. The free part $F$ of $H$ above is a retract of $H$ with normal complement $N$, the normal subgroup of $H$ generated by $S$. In particular, if $H$ is generated by its intersections with conjugates of $A$ and $B$, then $F=1$ and $H$ is the tree product $S$.

Proof. Since $H$ is an HNN group with base $S$, the first assertion follows.

Suppose $H$ is generated by its intersections with conjugates of $A$ and $B$. Since $A_{H}^{W}=A_{H}^{h D_{\alpha} a}=h A_{H}^{D a} h^{-1}<N$, and similarly $B_{H}^{W}<N$, we have $H=N$; hence $F=1$, and so $H=\operatorname{gp}(F, S)=S$. 
Corollary. Let $G, A, B, U, H$ be as above. Then the rank of the free part $F$ of $H$ is

$$
G:(H, U)-G:(H, A)-G:(H, B)+1
$$

where $G:(H, X)$ is the number of double cosets of $(H, X)$ in $G$.

Proof. The rank of $F$ is the number of $v$-double coset representatives $D_{\beta} E_{v}$ which are not $\alpha$ - or $\beta$-double coset representatives. Now every $\alpha$ - and $\beta$-double coset representative is a $v$-double coset representative, and only 1 is simultaneously an $\alpha$ - and a $\beta$-double coset representative.

Corollary. If $G, A, B, U, H$ are as above and $H$ intersects the conjugates of $U$ trivially, then $H$ is the free product of the free group $F$ and all the factors $A_{H^{\alpha}}^{D^{\alpha}}, B_{H^{\beta}}^{D}$.

The next corollary follows from the remarks on HNN groups preceding Lemma 2 of $\S 4$.

Corollary. Let $D_{\beta} E_{v}$ and $D_{\alpha} E_{u}$ be as in (3) above, and let $T_{D_{\beta} E_{v}}=\mathrm{gp}\left(t_{D_{\beta} E_{v}}, S\right)$. Then $T_{D_{B} E_{v}}$ is the HNN group

and

$$
\left\langle t_{D_{\beta} E_{v}}, S ; \text { rel } S, t_{D_{\beta} E_{v}} U_{H}^{D_{\alpha} E_{u}} t_{D_{\beta} E_{v}}^{-1}=U_{H^{\beta}}^{D^{2} E_{v}}\right\rangle,
$$

$$
H=\Pi^{*}\left(T_{D_{\beta} E_{v}} ; S\right)
$$

where $D_{\beta} E_{v}$ ranges over all $D_{\beta} E_{v}$ described in (1). Moreover, if we let $S_{D_{\beta} E_{v}}^{\prime}$ $=\mathrm{gp}\left(U_{H^{\alpha}}^{D E_{u}}, U_{H^{\beta}}^{D E_{v}}\right)$, then $T_{D_{\beta} E_{v}}$ is the free product of the HNN group

$$
T_{D_{\beta} E_{v}}^{\prime}=\left\langle t_{D_{\beta} E_{v}}, S_{D_{\beta} E_{v}}^{\prime} ; \text { rel } S_{D_{\beta} E_{v}}^{\prime}, t_{D_{\beta} E_{v}} U_{H^{\alpha} E_{u}}^{D_{D_{\beta} E_{v}}}=U_{H^{\beta} E_{v}}^{D^{\prime}}\right\rangle
$$

and $S$ with their common subgroup $S_{D_{\beta} E_{v}}^{\prime}$ amalgamated.

7. Indecomposable subgroups and subgroups satisfying an identity.

TheOREM 6. Let $G=(A * B ; U)$. Then an indecomposable subgroup $H$ of $G$ is either

(1) a subgroup of a conjugate of $A$ or $B$,

(2) a countable ascending union $U_{H}^{D_{i}}$ where each $D_{i}(i=0,1,2, \ldots)$ is an $\alpha$-or $\beta$-double coset representative and is obtained from $D_{i+1}$ by deleting the last syllable of $D_{i+1}$, or

(3) an HNN group of the form

$$
T^{\prime}=\left\langle t, S^{\prime} ; \operatorname{rel} S^{\prime}, t U_{H}^{\delta} t^{-1}=U_{H}^{\delta^{\prime}}\right\rangle
$$

where $\delta$ and $\delta^{\prime}$ are distinct $u$ - and $v$-double coset representatives for the same $(H, U)$ coset, $S^{\prime}=\mathrm{gp}\left(U_{H}^{\delta}, U_{H}^{\delta^{\prime}}\right)$, and $t=\delta^{\prime} P \delta^{-1}$ with $P$ in $U$.

Proof. We apply Theorem 5 to $H$.

Suppose first that each $t_{D_{\beta} E_{v}}=1$. Then $H$ is the tree product $S$. Since $S$ is indecomposable and has two vertices of level zero, we may infer from Theorem 2 
that $H$ is either a vertex of $S$, or $H$ is the countable ascending union of amalgamated subgroups corresponding to an infinite path determined by distinct vertices of increasing levels. This yields possibilities (1) and (2).

Suppose the free part of $H$ is nontrivial; then by the last corollary to Theorem 5 , $H=\Pi^{*}\left(T_{D_{\beta} E_{v}} ; S\right)$. Since $H$ is indecomposable, all factors $T_{D_{\beta} E_{v}}$ except one must equal $S$; hence $H=T_{D_{\beta} E_{v}}$ for some $D_{\beta} E_{v}$. Now $T_{D_{\beta} E_{v}}=\left(T_{D_{\beta} E_{v}}^{\prime} * S ; S_{D_{\beta} E_{v}}^{\prime}\right)$. Since $T_{D_{\beta} E_{v}}$ is indecomposable, $H=T_{D_{\beta} E_{v}}^{\prime}$ which by the last corollary to Theorem 5 has the form alledged in (3) above.

COROllary. If $U$ is countable, then any noncountable indecomposable subgroup $H$ of $G=(A * B ; U)$ is in a conjugate of a factor.

Corollary (Moldavanski). Any abelian subgroup $H$ of $G$ has the form (1), (2), or is the direct product of an infinite cyclic group and a subgroup of a conjugate of $U$.

Proof. An abelian subgroup is, of course, indecomposable. It therefore suffices to show that (3) reduces to a direct product. If $T^{\prime}$ in (3) is abelian, then $U_{H}^{\delta}=U_{H}^{\delta^{\prime}}$ $=S^{\prime}$. Hence by Tietze transformations $T^{\prime}$ is the direct product of $\mathrm{gp}(t)$ and $U_{H}^{\delta^{\prime}}$.

TheOREM 7. Let $G=(A * B ; U)$. Suppose $H$ is a subgroup of $G$ satisfying a nontrivial identity. Then $H$ is one of the following:

(1) a subgroup of a conjugate of $A$ or $B$;

(2) an ascending union as in (2) of Theorem 6;

(3) an HNN group as in (3) of Theorem 6 where $U_{H}^{\delta}<U_{H}^{\delta^{\prime}}$ or vice versa, and therefore $H$ is an infinite cyclic extension of $U_{H}^{\delta^{\prime}}, U_{H}^{\delta}$, or of a proper ascending union $\left\{U_{H}^{t^{k} \delta^{\prime}}\right\}$ or $\left\{U_{H}^{t^{-k} \delta}\right\}(k=0,1,2, \ldots)$;

(4) an amalgamated product

$$
\begin{aligned}
& \left(A_{H^{\alpha}}^{D^{\alpha}} * B_{H^{\beta}}^{D} ; U_{H}^{D}\right), \quad D=D_{\alpha} \text { or } D_{\beta}, \\
& \left(A_{H^{\alpha}}^{D^{\alpha}} * A_{H}^{D_{\alpha}^{\prime}} ; U_{H}^{D}\right), \quad D=D_{\alpha} \text { or } D_{\alpha}^{\prime},
\end{aligned}
$$

or

$$
\left(B_{H}^{D_{\beta}} * B_{H}^{D_{\beta}^{\prime}} ; U_{H}^{D}\right), \quad D=D_{\beta} \quad \text { or } \quad D_{\beta}^{\prime},
$$

and, moreover, $U_{H}^{D}$ is of index two in both factors of $H$.

Proof. If $H$ satisfies a nontrivial identity, then the free part $F$ of $H$ generated by the $t$ 's must be infinite cyclic or trivial. Suppose first $F$ is trivial. Then $H=S$, a tree product. Hence by Theorem 3 possibilities (1), (2) or (4) hold.

Suppose then $F$ is infinite cyclic. In that case, $H=T_{D_{\beta} E_{v}}$ (as defined in the last Corollary to Theorem 5), which equals $\left(T_{D_{\beta} E_{v}}^{\prime} * S ; S_{D_{\beta} E_{v}}^{\prime}\right)$. Since $S_{D_{\beta} E_{v}}^{\prime}$ has infinite index in $T_{D_{\beta} E_{v}}^{\prime}$, we have that $H=T_{D_{\beta} E_{v}}^{\prime}$. Now it is easy to see from Lemma 2 applied to the HNN group $T^{\prime}=T_{D_{\beta} E_{v}}^{\prime}$ that the normal subgroup $N$ of $T^{\prime}$ generated by $S^{\prime}=S_{D_{\beta} E_{v}}^{\prime}$ is the tree product of the groups $S_{k}^{\prime}=t^{k} S^{\prime} t^{-k}$ (where $t=t_{D_{\beta} E_{v}}$ and $k$ is any integer), where the factors $S_{k}^{\prime}$ and $S_{k+1}^{\prime}$ are neighbors with the subgroups 
$U_{H}^{t^{k} \delta^{\prime}}=U_{H}^{t^{k+1 \delta}}$ amalgamated. As in the proof of Lemma 2, we may assign $|k|$ as the level of $S_{k}^{\prime}$. Since $N$ satisfies a nontrivial identity, $N=S_{k}^{\prime}$, or $N$ is the ascending union $S_{0}^{\prime}<S_{\varepsilon}^{\prime}<S_{2 \varepsilon}^{\prime}<\cdots(\varepsilon= \pm 1)$, or $N=\operatorname{gp}\left(S_{k}^{\prime}, S_{m}^{\prime}\right)$ with $k<m$. Since $N$ is normal, it follows that $S_{0}^{\prime}<S_{1}^{\prime}$ or $S_{0}^{\prime}<S_{-1}^{\prime}$. This is immediate if either of the first two possibilities on $N$ holds; the third possibility implies the first. For, if $N=\operatorname{gp}\left(S_{k}^{\prime}, S_{m}^{\prime}\right)$, then $N$ is the tree product of $S_{k}^{\prime}, S_{k+1}^{\prime}, \ldots, S_{m}^{\prime}$ which is the tree product of $S_{0}^{\prime}$, $S_{1}^{\prime}, \ldots, S_{m-k}^{\prime}$ which is the tree product of $S_{k-m-1}^{\prime}, \ldots, S_{-1}^{\prime}$; but the intersection of these last two tree products is $U_{H}^{\delta}$ and so $N=S_{0}^{\prime}$.

Suppose $S_{0}^{\prime}<S_{1}^{\prime}$. Then $S_{0}^{\prime}=U_{H}^{\delta^{\prime}}$, so that $U_{H}^{\delta}<U_{H}^{\delta^{\prime}}$; moreover, $S_{k}^{\prime}=U_{H}^{t^{k} \delta^{\prime}}$. If $S_{0}^{\prime}<S_{-1}^{\prime}$, interchange $\delta$ and $\delta^{\prime}$. Hence possibility (3) holds.

\section{Finitely generated subgroups.}

TheOREM 8. Suppose $G=(A * B ; U)$ where $A, B$ have the property that their finitely generated subgroups are finitely related, and $U$ has the property that each of its subgroups is finitely generated. Then all finitely generated subgroups of $G$ are finitely related.

Proof. Let $H$ be a finitely generated subgroup of $G$. Now by Theorem $5, H$ is an HNN group with base $S$ and associated subgroups which are intersections of $H$ with conjugates of $U$. Hence these associated subgroups of $H$ are finitely generated. Therefore by Lemma 3, the free part of $H$ and $S$ are both finitely generated. Moreover, $S$ is a tree product whose vertices are subgroups of conjugates of $A, B$ and whose amalgamated subgroups are subgroups of conjugates of $U$; hence $S$ is the tree product of finitely many of its vertices each of which is finitely generated. But finitely generated subgroups of $A$ or $B$ are finitely related; therefore $S$ is finitely related, and so $H$ is finitely related.

Corollary. Let $G=(A * B ; U)$ where $A, B$ are as above, and suppose that $H$ is a finitely generated subgroup of $G$ whose intersection with each conjugate of $U$ is finitely generated. Then $H$ is a finitely related group.

Proof. Since the associated subgroups of $H$ and the amalgamated subgroups of $S$ in the above argument are intersections of $H$ with conjugates of $U$, the result follows as above.

If $A, B$ are as in Theorem 8 , but $U$ does not satisfy the maximal condition, then $(A * B ; U)$ may have finitely generated subgroups which are not finitely related. For example, let

$$
A=\left\langle x, y ; x^{2}=y^{3}\right\rangle, \quad B=A_{1}=\left\langle x_{1}, y_{1} ; x_{1}^{2}=y_{1}^{3}\right\rangle,
$$

let $U$ be the commutator subgroup of $A$, which is a free group generated by $x y x^{-1} y^{-1}$ and $x y^{2} x^{-1} y^{-2}$, and let $K$ be the free subgroup of $A$ freely generated by $y x y$ and $x y x y x^{-1}$; similarly, let $U_{1}$ and $K_{1}$ be the corresponding subgroups of $A_{1}$. Now by Theorem 8 , the finitely generated subgroups of $A$ and $A_{1}$ are finitely related. On the other hand, the subgroup $H$ of $\left(A * A_{1} ; U=U_{1}\right)$ generated by $K$ 
and $K_{1}$ is $\left(K * K_{1} ; U \cap K=U_{1} \cap K_{1}\right)$ (see [11, p. 512]), which is finitely generated. However, $H$ is not finitely related; to show this it suffices to show that $U \cap K$ is infinitely generated (see G. Baumslag [2]). Now $U \cap K$ is a nontrivial normal subgroup of the free group $K ; U \cap K$ is of infinite index in $K$ since $K /(U \cap K)$ $\cong U K / U$, which is an infinite cyclic subgroup of $A / U$; hence, $U \cap K$ is infinitely generated (see, e.g., [9, p. 104]).

TheOrem 9. Let $G=(A * B ; U)$ where $A, B$ are locally indicable and $U$ is cyclic. Then $G$ is locally indicable.

Proof. We first observe that if $A, B$ are indicable, then $G$ is indicable. For, if $A, B$ can be mapped homomorphically onto the infinite cyclic groups generated by $x$ and $y$, respectively, then $G$ can be mapped homomorphically onto the group

$$
\left\langle x, y ; x^{r}=y^{s}\right\rangle
$$

for suitable $r, s$. This group in turn can be mapped homomorphically onto an infinite cyclic group: namely, if $s=0$, map $x \rightarrow 1, y \rightarrow y$, and otherwise map $x \rightarrow y^{s}, y \rightarrow y^{r}$.

Suppose now $A, B$ are locally indicable, and $H$ is a finitely generated subgroup of $G$. If the free part of $H$ is nontrivial, $H$ is clearly indicable. Otherwise, $H$ is the tree product $S$ of Theorem 5. By Theorem 4 above, $H$ is the tree product of finitely many finitely generated subgroups of conjugates of $A$ or $B$ with cyclic amalgamated subgroups. Therefore $H$ is the tree product of finitely many indicable groups with cyclic amalgamated subgroups. A simple inductive argument using the first remark shows that $H$ is indicable.

If $A, B$ are locally indicable but the amalgamated subgroup $U$ is not cyclic, then $(A * B ; U)$ need not be locally indicable. For example, let

and

$$
A=\left\langle x, y ; x y x^{-1}=y^{-1}\right\rangle
$$

$$
B=\left\langle z, w ; z w z^{-1}=w^{-1}\right\rangle .
$$

Then $A, B$ are locally indicable (since they are locally indicable extensions of locally indicable groups). Moreover, the torsion free subgroups

$$
U=\operatorname{gp}\left(x^{2}, y\right), \quad V=\operatorname{gp}\left(w, z^{2}\right)
$$

are each free abelian of rank two, and so we may form the amalgamated product $G=\left(A * B ; x^{2}=w, y=z^{2}\right) . G$ is not indicable, since the commutator quotient group is finite.

In this counterexample, $U$ is free abelian of rank two. A counterexample in which $U$ is a free group of rank two is provided by taking

$$
\begin{aligned}
A & =\left\langle x, y ; x y x^{-1}=y^{-1}\right\rangle *_{y=z}\left\langle z, w ; z w z^{-1}=w^{-1}\right\rangle, \\
B & =\left\langle p, q ; p q p^{-1}=q^{-1}\right\rangle *_{q=r}\left\langle r, s ; r s r^{-1}=s^{-1}\right\rangle,
\end{aligned}
$$

and $G=(A * B ; x=s, w=p)$. 
The following definition and lemma generalize corresponding ones for free products (see B. Baumslag [1]).

Definition. Let $G=(A * B ; U)$. Then a set of words in generators of $A$ and $B$ is double ended if there exist two words in the set whose $\alpha$ - and $\beta$-syllables do not define elements of $U$, and such that one of the two words ends in an $\alpha$-syllable and the other ends in a $\beta$-syllable. A set of elements of $G$ is double ended if there exists a double ended set of words defining these elements.

Lemma 8. Let $G, A, B, U, H, F, S$ be as in Theorem 5. Then the number of double ended $(H, U)$ cosets in $G$ is finite iff $F$ is finitely generated and $S$ is the tree product of finitely many of its vertices.

Proof. Clearly $F$ is finitely generated iff the number of $D_{\beta} E_{v}$ such that $t_{D_{\beta} E_{v}} \neq 1$ is finite.

Moreover, $S$ is the tree product of finitely many of its vertices iff the number of double coset representatives $D_{\alpha}, D_{\beta}$ such that $A_{H^{\alpha}}^{D^{\alpha}} \neq U_{H^{\alpha}}^{D^{\alpha}}$ or $B_{H^{\beta}}^{D^{\beta}} \neq U_{H^{\beta}}^{D^{\beta}}$ is finite. For, if the number of such $D_{\alpha}$ and $D_{\beta}$ is finite, then the set $J$ of corresponding vertices together with the vertices of level zero is finite. Using induction on the level of a vertex of $S$, it is easy to see that every vertex of $S$ is a subgroup of a vertex in $J$, so that $S$ is the tree product of finitely many of its vertices. Conversely, if $S$ is the tree product of finitely many of its vertices including the vertices of level zero, then by Theorem 1 each vertex not in this finite subtree is equal to the amalgamated subgroup corresponding to the first edge in a path joining the vertex to the subtree. But if a vertex $A_{H^{\alpha}}^{D}$ or $B_{H^{\beta}}^{D}$ is not in the finite subtree, then the first edge on a path joining the vertex to a vertex of level zero corresponds to $U_{H^{\alpha}}^{D^{\alpha}}$ or $U_{H^{\beta}}^{D^{\beta}}$, respectively. Hence, there are only finitely many $D_{\alpha}$ and $D_{\beta}$ such that $A_{H^{\alpha}}^{D^{\alpha}} \neq U_{H^{\alpha}}^{D^{\alpha}}$ or $B_{H^{\beta}}^{D} \neq U_{H^{\beta}}^{D}$.

(i) Suppose now there are only finitely many $(H, U)$ cosets which are double ended. We first show that the number of $D_{\beta} E_{v}$ such that $t_{D_{\beta} E_{v}} \neq 1$ is finite by showing that if $t_{D_{\beta} E_{v}} \neq 1$, then $H D_{\beta} E_{v} U$ is double ended. For, clearly $E_{v} \neq 1$. Moreover, if ${ }^{\alpha}\left(D_{\beta} E_{v}\right)=D_{\alpha} E_{u} P$, then $E_{u} \neq 1$; otherwise

$$
D_{\beta} E_{v}={ }^{v}\left|D_{\beta} E_{v}\right|={ }^{v}\left|D_{\alpha} P\right|={ }^{v}\left|D_{\alpha}\right|=D_{\alpha},
$$

contrary to $D_{\beta} E_{v}$ not being an $\alpha$-representative (where ${ }^{v}|W|$ is the $v$-double coset representative of $W$ ). Thus the two words $D_{\beta} E_{v}$ and $D_{\alpha} E_{u} P$ end in a $\beta$ - and in an $\alpha$-syllable, respectively. Since different $D_{\beta} E_{v}$ determine different $(H, U)$ cosets, the number of $D_{\beta} E_{v}$ such that $t_{D_{B} E_{v}} \neq 1$ is finite, so that $F$ is finitely generated.

Next consider those $D_{\alpha}$ and $D_{\beta}$ such that $A_{H^{\alpha}}^{D^{\alpha}} \neq U_{H^{\alpha}}^{D^{\alpha}}$ or $B_{H^{\beta}}^{D^{\beta}} \neq U_{H^{\beta}}^{D^{\beta}}$. Suppose $A_{H^{\alpha}}^{D} \neq U_{H^{\alpha}}^{D}, D_{\alpha} \neq 1$. Then there exists $a \in A-U$ such that $D_{\alpha} a D_{\alpha}^{-1} \in H$. Clearly, $H D_{\alpha} U=H D_{\alpha} a U$, and the pair $D_{\alpha}, D_{\alpha} a$ end in opposite type syllables, so that $H D_{\alpha} U$ is double ended. Similarly, if $B_{H^{\beta}}^{D^{\beta}} \neq U_{H^{\beta}}^{D}, D_{\beta} \neq 1$, then $H D_{\beta} U$ is double ended. But different $D_{\alpha}, D_{\beta}$ determine different $(H, U)$ cosets; hence, there are 
only finitely many such $D_{\alpha}$ and $D_{\beta}$. Consequently, $S$ is the tree product of finitely many of its vertices.

(ii) Conversely, suppose that (1) the number of $D_{\beta} E_{v}$ such that $t_{D_{\beta} E_{v}} \neq 1$ is finite and (2) the number of $D_{\alpha}$ and $D_{\beta}$ such that $A_{H^{\alpha}}^{D^{\alpha}} \neq U_{H^{\alpha}}^{D^{\alpha}}$ or $B_{H^{\beta}}^{D^{\prime}} \neq U_{H^{\beta}}^{D^{\beta}}$ is finite.

If a double coset $H W U$ is double ended we show that ${ }^{v}|W|$ is an initial segment of the $\alpha$ - or $\beta$-representative of some $D_{\beta} E_{v}$ in (1) or is an initial segment of some $D_{\alpha}$ or $D_{\beta}$ in (2). For suppose otherwise. If $H W U$ is double ended, then there exists $h \in H$ such that ${ }^{v}|W|$ and $h^{v}|W|$ end in opposite type syllables. Choose $W$ and $h$ so that ${ }^{v}|W|$ is not an initial segment as claimed, ${ }^{v}|W|$ and $h \cdot v|W|$ end in opposite type syllables, and $h \cdot v|W|$ has minimum length (as an element of $(A * B ; U)$ ) subject to the preceding two conditions.

Clearly, $v|W| \neq 1$ and $t_{v_{|w|}}=1$. Therefore ${ }^{v}|W|$ is either an $\alpha$-double coset representative $D_{\alpha}$ or a $\beta$-double coset representative $D_{\beta}$. Assume ${ }^{v}|W|=D_{\alpha}$ (an analogous argument can be used if $v|W|=D_{\beta}$ ). Then $v|W|$ ends in a $\beta$-syllable, and the element $h \cdot v|W|$ ends in an $\alpha$-syllable. Therefore

$$
h^{-1}=D_{\alpha}\left(h D_{\alpha}\right)^{-1}=D_{\alpha} a L
$$

where $a \in A-U$ and $L$ begins with a $\beta$-syllable $\left(L \neq 1\right.$, since otherwise $D_{\alpha} a \in H$ and so $\left.D_{\alpha}={ }^{\alpha}\left|D_{\alpha} a\right|=1\right)$. Thus $h^{-1}=D_{\alpha} a b M$ where $b \in B-U$, and $M=1$ or $M$ begins with an $\alpha$-syllable. Let ${ }^{\alpha}\left(D_{\alpha} a\right)=D_{\alpha} E_{u} P$. Then

$$
h^{-1}=D_{\alpha} a \cdot P^{-1} E_{u}^{-1} D_{\alpha}^{-1} \cdot D_{\alpha} E_{u} b^{\prime} M
$$

where $b^{\prime}=P b$. Since $A_{H^{\alpha}}^{D}=U_{H^{\alpha}}^{D}$,

$$
h^{-1}=D_{\alpha} u D_{\alpha}^{-1} \cdot D_{\alpha} E_{u} b^{\prime} M=D_{\alpha} u E_{u} b^{\prime} M
$$

where $u \in U$. Since the length of $h^{-1}$ is $\lambda\left(D_{\alpha}\right)+2+\lambda(M)$ (where " $\lambda$ " denotes the length of an element in $(A * B ; U))$, it follows that $E_{u} \neq 1$. Let $k^{-1}=D_{\alpha} E_{u} b^{\prime} M$; then $k \in H$. We show that $D_{\alpha} E_{u}$ is a $\beta$-double coset representative $D_{\beta}$; it would then follow that $D_{\beta}$ and $k D_{\beta}$ end in opposite type syllables, $D_{\beta}$ is not an initial segment of an $\alpha$ - or $\beta$-representative of a representative in (1) or (2) (for otherwise, $D_{\alpha}$ would be such an initial segment), and yet $k D_{\beta}$ has smaller length than $h D_{\alpha}$, contrary to the minimality assumption on the length of $h D_{\alpha}$.

To show $D_{\alpha} E_{u}$ is a $\beta$-double coset representive, let ${ }^{v}\left|D_{\alpha} E_{u}\right|=D_{\beta} E_{v}$. Then $t_{D_{\beta} E_{v}}=1$, since $D_{\alpha}$ is an initial segment of ${ }^{\alpha}\left(D_{\beta} E_{v}\right)$; indeed ${ }^{\alpha}\left(D_{\beta} E_{v}\right)={ }^{u}\left|D_{\beta} E_{v}\right| P^{\prime}$ $=D_{\alpha} E_{u} P^{\prime}$ where $P^{\prime} \in U$. Thus $D_{\beta} E_{v}=D_{\alpha} E_{u} P^{\prime}$; since $E_{u} \neq 1, E_{v}$ and $P^{\prime}$ must equal 1, and hence $D_{\beta}=D_{\alpha} E_{u}$.

Consequently, the number of double ended $(H, U)$ cosets is finite.

Corollary. Suppose $G=(A * B ; U)$, and $H$ is a finitely generated subgroup of $G$. Then the number of double ended $(H, U)$ cosets in $G$ is finite.

Proof. Since $H$ is finitely generated, its free part $F$ is also finitely generated. Moreover, each of the associated subgroups of the HNN group $H$ as given in 
Theorem 5 is contained in a vertex of the tree product $S$, the base of $H$. Hence by Lemma $3, S$ is the tree product of finitely many of its vertices. Therefore by the preceding lemma, $(H, U)$ has finitely many double ended cosets in $G$.

THEOREM 10. Let $G=(A * B ; U)$ and let $H$ be a finitely generated subgroup of $G$ containing a normal subgroup $N$ of $G, N \nless U$. Then $(H, U)$ is of finite index in $G$. Moreover, $H$ is of finite index in $G$ iff the intersection of $U$ with each conjugate of $H$ is of finite index in $U$; in particular, if $U$ is finite, then $H$ has finite index in $G$.

Proof. Assume that $U$ is not of index two in both $A$ and $B$. We show that each $(H, U)$ coset is double ended; since $H$ is finitely generated the preceding corollary would then show that $(H, U)$ has finite index in $G$.

Now it is easy to show that $N$ contains an element of $G$ which begins and ends with an $\alpha$-syllable and an element which begins and ends with a $\beta$-syllable. Then each double coset $H w U$ with $w \notin U$ can be written $H w x U$ where $x \in N$ and $w, x$ end in opposite type syllables; hence, $H w U$ is double ended.

On the other hand, if $U$ is of index two in both $A$ and in $B$, then the index of $(H, U)$ in $G$ is the index of $H U / U$ in $G / U=(A / U) *(B / U)$, the infinite dehedral group. Moreover, $H U / U$ contains the nontrivial subgroup $N U / U$ which is normal in $G / U$ and hence of finite index in $G / U$.

Finally, since the number of $H$ cosets in $H w U$ is the index of $U \cap w^{-1} H w$ in $U$, the last part of the theorem follows.

Corollary. Suppose $G=(A * B ; U)$ where $A, B$ are free and $U$ is cyclic. If $H$ is finitely generated and contains a normal subgroup $N$ of $G$ where $N$ is not free, then $H$ is of finite index in $G$; in particular, a finitely generated normal subgroup of $G$ is either free or of finite index in $G$.

Proof. $N$ intersects $U$ nontrivially; for otherwise by the third corollary to Theorem 5, $N$ would be a free product of free groups and hence free. Therefore each conjugate of $H$ intersects $U$ nontrivially and the intersection is of finite index in the cyclic group $U$.

THEOREM 11. Let $G=(A * B ; U)$. If $A, B$ have the finitely generated intersection property, and $U$ is finite, then $G$ has the finitely generated intersection property.

Proof. Let $H, K$ be finitely generated subgroups of $G$. Then the number of double ended cosets of $(H, U)$ and of $(K, U)$ in $G$ is finite. Since $U$ is finite, the intersection of an $(H, U)$ coset with a $(K, U)$ coset contains only finitely many $(H \cap K, U)$ cosets; hence there are only finitely many double ended $(H \cap K, U)$ cosets in $G$. Therefore by Lemma $8, F$ is finitely generated and $S$ is generated by finitely many of its vertices.

Moreover, applying Lemma 3 and Theorem 4 to $H$ (given as an HNN group as in Theorem 5), we see that $X_{H}^{D}$ is finitely generated where $X$ is $A$ or $B$ and $D$ 
is a double coset representative; hence the intersection of $H$ with any conjugate of $A$ or $B$ is finitely generated. Similarly, the intersection of $K$ with any conjugate of $A$ or $B$ is finitely generated. Since $A, B$ have the finitely generated intersection property, the intersection of $H \cap K$ with any conjugate of $A$ or $B$ is finitely generated.

Consequently, $H \cap K$ is finitely generated.

The following corollary can be proved using a similar argument.

Corollary. Let $G=(A * B ; U)$ where $A, B$ are as above, and $U$ is finitely generated. Let $H, K$ be finitely generated subgroups of $G$ with the property that the intersection of each conjugate of $H$ with $U$ and of each conjugate of $K$ with $U$ is of finite index in $U$. Then $H \cap K$ is finitely generated.

The group $G=\left\langle x, y ; x^{2}=y^{3}\right\rangle$ shows that even if the factors and the amalgamated subgroup are cyclic, a finitely generated $N \triangleleft G, N \nless U$, need not be of finite index in $G$, and the intersection of two finitely generated subgroups $H, K$ need not be finitely generated. Specifically, let $N=H=\mathrm{gp}\left(x y x^{-1} y^{-1}, x y^{2} x^{-1} y^{-2}\right)$ $=$ the commutator subgroup of $G$, and let $K=\mathrm{gp}\left(y x y, x y x y x^{-1}\right)$. As in the example following the corollary to Theorem $8, H \cap K$ is infinitely generated; moreover, the commutator quotient group $G / N$ is infinite cyclic.

\section{REFERENCES}

1. B. Baumslag, Intersections of finitely generated subgroups in free products, J. London Math. Soc. 41 (1966), 673-679. MR 33 \#7396.

2. G. Baumslag, A remark on generalized free products, Proc. Amer. Math. Soc. 13 (1962), 53-54. MR 24 \#A3191.

3. - On generalized free products, Math. Z. 78 (1962), 423-428. MR 25 \#3980.

4. G. Higman, The units of group-rings, Proc. London Math. Soc. (2) 46 (1940), 231-248. MR 2, 5.

5. - Subgroups of finitely presented groups, Proc. Roy. Soc. London Ser. A 262 (1961), 455-475. MR 24 \#A152.

6. G. Higman, B. H. Neumann and H. Neumann, Embedding theorems for groups, J. London Math. Soc. 24 (1949), 247-254. MR 11, 322.

7. A. G. Howson, On the intersection of finitely generated free groups, J. London Math. Soc. 29 (1954), 428-434. MR 16, 444.

8. A. Kuroš, The theory of groups. Vol. 2, GITTL, Moscow, 1953; English transl., Chelsea, New York, 1956. MR 15, 501; MR 18, 188.

9. W. Magnus, A. Karrass and D. Solitar, Combinatorial group theory: Presentations of groups in terms of generators and relations, Pure and Appl. Math., vol. 13, Interscience, New York, 1966. MR 34 \#7617.

10. D. I. Moldavanski, Certain subgroups of groups with one defining relation, Sibirsk. Mat. $\check{Z}$. 8 (1967), 1370-1384. MR 36 \#3862.

11. B. H. Neumann, An essay on free products with amalgamations, Philos. Trans. Roy. Soc. London Ser. A. 246 (1954), 503-544. MR 16, 10.

12. H. Neumann, Generalized free products with amalgamated subgroups, Amer. J. Math. 70 (1948), 590-625. MR 10, 233. 
13. H. Newmann, Generalized free products with amalgamäted subgroups. II, Amer. J. Math. 71 (1949), 491-540. MR 11, 8.

14. O. Schreier, Die Untergruppen der freien Gruppen, Abh. Math. Sem. Univ. Hamburg 5 (1927), 161-183.

15. A. J. Weir, The Reidemeister-Schreier and Kuroš subgroup theorems, Mathematika 3 (1956), 47-55. MR 18, 280.

YORK UNIVERSITY,

Toronto, CANADA 